\title{
Séchage des lactosérums et dérivés : rôle du lactose et de la dynamique de l'eau
}

\author{
Pierre SCHUCK ${ }^{\mathrm{a} *}$, Said BouhaLlab ${ }^{\mathrm{a}}$, Delphine Durupt ${ }^{\mathrm{b}}$, \\ Philippe VAREILLE ${ }^{b}$, Jean-Paul HUMBERT ${ }^{b}$, Michèle MARIN $^{c}$ \\ ${ }^{\text {a }}$ UMR Science et Technologie du Lait et de l'Oeuf, INRA-Agrocampus, 65, rue de St-Brieuc, \\ 35042 Rennes Cedex, France \\ b Eurosérum, BP 17, 70170 Port sur Saône, France \\ ${ }^{c}$ UMR de Génie et Microbiologie des Procédés Alimentaires, INRA - INA P-G, CBAI, \\ 78850 Thiverval-Grignon, France
}

Received 21 February 2003 - Accepted 10 December 2003

Published online 26 March 2004

\begin{abstract}
Spray drying of wheys and derivatives. Spray drying is one of the most convenient technique for producing conventional milk powders. However, its use for the dehydration of new and diversified products, such as whey and derivatives, is rather empirical and generally limited by the appearance of several problems such as the sticking and the caking of generated powders. The origin of these problems is linked to complex interactions between several factors: physical properties of the product (viscosity, relative humidity and hygroscopicity), its composition (mineral, protein and carbohydrate contents and $\mathrm{pH}$ ) and process parameters (outlet and inlet temperatures, heating and cooling rates and agitation). This review paper highlights the research works that deal with the impact of these factors on spray drying. In particular, we focus on how these factors affect water activity, glass transition and lactose crystallization, the main important variable to be controlled for better optimization of spray drying process of whey derivative products.
\end{abstract}

\section{Lactose / water / crystallization / spray drying / whey}

Résumé - Le séchage par atomisation s'est imposé à l'échelle industrielle pour le traitement des produits laitiers conventionnels. Son adaptation à la déshydratation des produits nouveaux et diversifiés, tels que les lactosérums et perméats, se fait de manière empirique et pose un certain nombre de problèmes, en particulier le collage des produits en tour de séchage et leur mottage à l'état de poudre. Les facteurs à l'origine de ces deux phénomènes sont multiples et interdépendants : propriétés physiques des produits (viscosité, $\mathrm{a}_{\mathrm{w}}$ et hygroscopicité), composition des produits (teneur et nature minérale et protéique, forme du lactose et $\mathrm{pH}$ ) et paramètres du procédé (températures d'entrée et de sortie, vitesses de chauffage et de refroidissement et agitation). Le lien entre ces différents facteurs est traité dans cette revue au travers de leur impact sur la dynamique de l'eau et, par conséquent, sur la cristallisation du lactose. Les connaissances actuelles dans ce domaine dérivent des résultats obtenus sur des mélanges « modèles ». Des travaux sur produits complexes, prenant en compte l'interdépendance de ces différents facteurs sont indispensables pour prédire et maîtriser les conditions permettant d'obtenir une poudre de bonne stabilité.

\section{Lactose / eau / cristallisation / séchage par atomisation / lactosérum}

\footnotetext{
* Auteur correspondant : schuck@ rennes.inra.fr
} 


\section{INTRODUCTION}

Le lactosérum est un produit découvert il y a plus de 3000 ans avant Jésus-Christ, par des Bédouins lors du transport de lait : l'acidification et la coagulation par la chaleur provoquaient la formation d'une phase liquide au-dessus d'un caillé de lait [19]. Pendant de nombreuses années, le lactosérum ou petit lait a été considéré comme un déchet encombrant, un sous produit des fromageries et caséineries dont l'utilisation, lorsqu'elle en était faite, se limitait à l'alimentation animale et à la fertilisation des champs [95].

Le lactosérum est cependant un produit intéressant par ses teneurs en protéines riches en acides aminés indispensables (lysine et tryptophane), en lactose et par la présence de nombreuses vitamines du groupe $B$ comme la thiamine et la riboflavine [101].

La production de lactosérum correspond à 9 fois le tonnage de fromages fabriqués [95]. En conséquence, une industrie de traitement du lactosérum s'est donc considérablement développée ces dernières décennies. Ce développement s'est fait pour des raisons économiques d'une part - les industriels laitiers désirant la valorisation de ce co-produit - et pour des raisons écologiques d'autre part [11]. Toutes ces raisons ainsi que la mise en place de quotas laitiers et du développement de nouvelles technologies permettant de fractionner les différents composés du lait ont contribué au développement de nouveaux produits secs et stables [87]. En France, la production de poudre de lactosérum a doublé en 15 ans passant de 329000 à 621000 tonnes en 2000 [10]. Les poudres de lactosérum et dérivés sont aujourd' hui très utilisés comme ingrédients non seulement pour leurs bonnes propriétés techno-fonctionnelles (industrie laitières, confiserie, charcuterie...) mais aussi nutritionnelles (élaboration d'aliments diététiques, pharmaceutiques ou infantiles) [19, 68].

Le séchage du lactosérum a pour but essentiel de stabiliser les produits par extraction de l'eau. L'amélioration des tech- nologies, les changements des habitudes alimentaires, la baisse des coûts, et une meilleure stabilité des poudres sont les principales raisons qui ont contribuées à l'accroissement de l'utilisation des produits sous forme déshydratée. La diversification des produits au niveau des compositions a cependant rendu l'opération de séchage plus délicate, moins routinière et les paramètres technologiques sont plus difficilement extrapolables d'un produit à un autre. Ainsi, des problèmes de collage en tour ou de mottage en silo sont fréquemment rencontrés conduisant à une diminution de la fonctionnalité, de la qualité et de la stabilité des poudres.

Dans cet article, nous décrivons successivement les rôles de la composition des produits, de la disponibilité de l'eau au cours du séchage, de la cristallisation du lactose ainsi que de la combinaison de ces différents facteurs sur les phénomènes de collage et de mottage.

\section{COMPOSITION DES LACTOSÉRUMS}

Le lactosérum, constitué essentiellement d'eau, contient du lactose, des protéines, des minéraux et un peu de matière grasse. La quantité et la proportion relative de ces différents constituants dépendent entre autres des procédés d'obtention. Selon le procédé, différents types de lactosérums peuvent ainsi être obtenus comme illustré sur la figure 1. Ces lactosérums peuvent être classés en deux principales catégories selon l'acidité du liquide obtenu [2] :

- Les lactosérums doux dont l'acidité varie entre 15 et $22^{\circ}$ Dornic $(\mathrm{pH} \approx 6,5)$. Ils sont issus de la production de pâtes pressées et/ou cuites (Edam, St Paulin, Emmental).

- Les lactosérums acides obtenus lors de la fabrication des pâtes fraîches et molles ou lors de la production des caséines atteignent $120^{\circ}$ Dornic, soit un $\mathrm{pH}$ proche de 4,5 .

Les lactosérums doux sont pauvres en calcium et phosphore contrairement aux lactosérums acides. De nombreux auteurs 


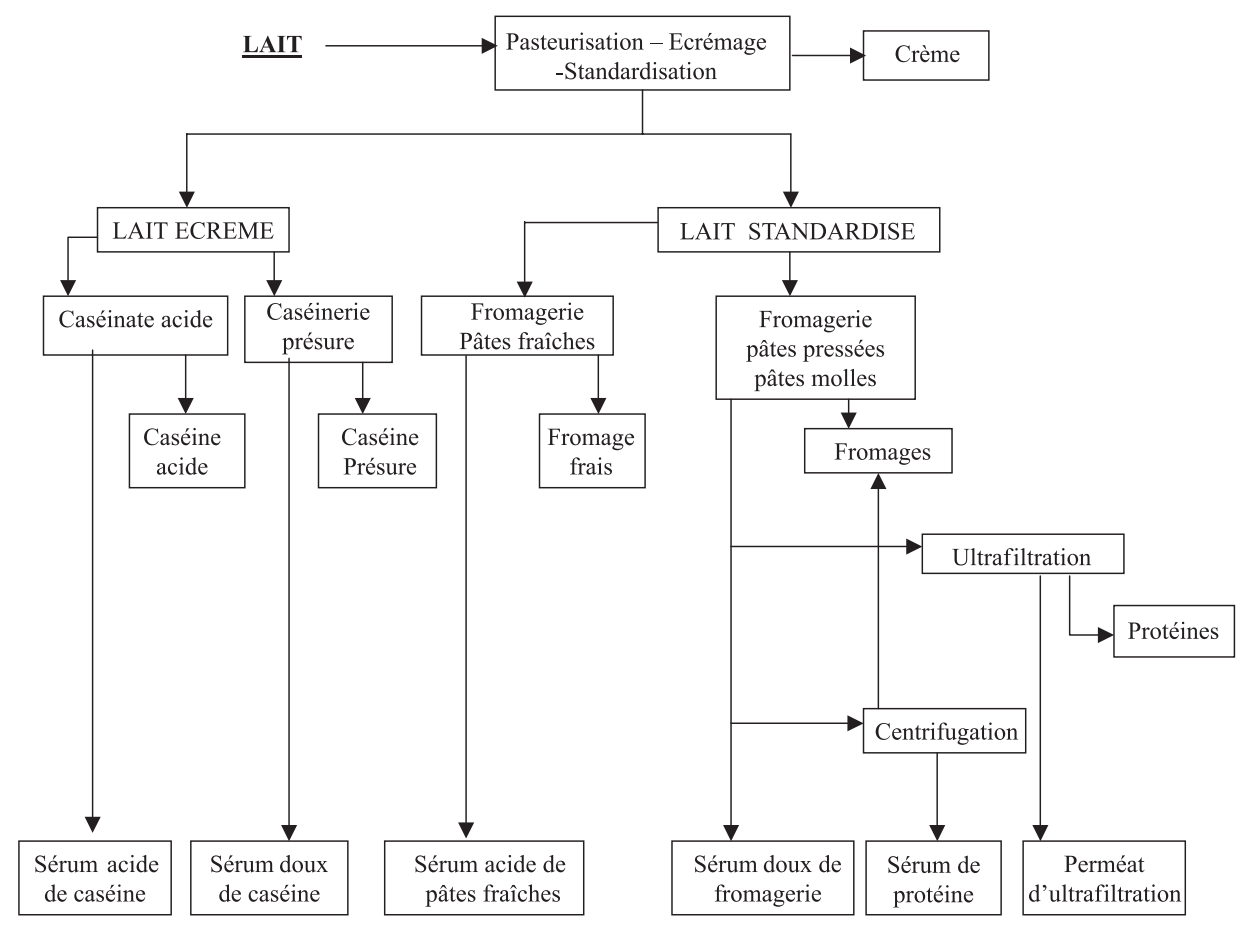

Figure 1. Voies technologiques permettant l'obtention des principaux types de lactosérums issus de la première transformation du lait [2].

Figure 1. Technological diagram of various whey after first transformation of milk [2].

ont déterminé la composition de ces produits $[29,44]$. Dans le tableau I sont rassemblées les compositions moyennes des deux catégories de lactosérums [91]. Le lactosérum de caséinerie est obtenu par la précipitation de la caséine par l'acide chlorhydrique ou sulfurique alors que le lactosérum acide est généré par une fermentation lactique d'un lait écrémé. Un lactosérum doux présente une teneur en protéines supérieure à celle d'un lactosérum acide en raison de la précipitation acide de certaines protéines. $\mathrm{La}$ différence majeure entre ces deux catégories de lactosérums se situe au niveau des teneurs en lactose et en acide lactique.

Les lactosérums doux sont plus facilement valorisables industriellement que les lactosérums acides qui posent plus de difficultés lors du séchage. Ces problèmes sont dus en partie à la forte minéralisation, à la faible teneur en lactose et à la variabilité de la composition des sérums acides [2, 85].

Dans les cas des lactosérums acides, du fait de la diversité des technologies de fabrication fromagère, une forte hétérogénéité peut être obtenue au niveau de la composition minérale entre pâtes fraîches et molles. À titre d'exemple, la composition minérale détaillée, anions et cations, déterminée par électrophorèse capillaire dans le cas des deux lactosérums acides est donnée dans le tableau II [85]. Il existe des variations significatives au niveau des teneurs en potassium, calcium, magnésium, phosphore, acétate et lactate. De plus, pour un même type, d'important coefficients de variation sont observés. D' autres études ont montré que la composition chimique et 
Tableau I. Composition des lactosérums doux et acides [91].

Table I. Composition of sweet and acid wheys [91].

\begin{tabular}{lcc}
\hline Constituants & $\begin{array}{c}\text { Sérum doux } \\
(\text { Cheddar })\end{array}$ & $\begin{array}{c}\text { Sérum acide lactique } \\
\text { (Fromage frais) }\end{array}$ \\
\hline Eau (\%) & 93,30 & 93,58 \\
Extrait sec (\%) & 6,70 & 6,42 \\
Azote total (g N·100 g $\left.{ }^{-1} \mathrm{MS}\right)$ & 1,94 & 1,85 \\
$\mathrm{NPN}\left(\mathrm{g} \mathrm{N} \cdot 100 \mathrm{~g}^{-1} \mathrm{MS}\right)$ & 0,51 & 0,53 \\
Protéines $\left(\mathrm{g} \mathrm{N} \cdot 100 \mathrm{~g}^{-1} \mathrm{MS}\right)$ & 1,43 & 1,32 \\
Lactose $\left(\mathrm{g} \cdot 100 \mathrm{~g}^{-1} \mathrm{MS}\right)$ & 74,62 & 68,53 \\
Cendres $\left(\mathrm{g} \cdot 100 \mathrm{~g}^{-1} \mathrm{MS}\right)$ & 7,76 & 9,34 \\
Acide lactique $\left(\mathrm{g} \cdot 100 \mathrm{~g}^{-1} \mathrm{MS}\right)$ & 2,09 & 7,32 \\
Acide citrique $\left(\mathrm{g} \cdot 100 \mathrm{~g}^{-1} \mathrm{MS}\right)$ & 1,49 & - \\
$\mathrm{pH}$ & 6,10 & 4,70 \\
Densité à $15{ }^{\circ} \mathrm{C}$ & 1,026 & $1,024-1,025$ \\
\hline
\end{tabular}

Tableau II. Composition anionique et cationique de deux groupes de lactosérums acides exprimée en $\%$ de la matière sèche [85].

Table II. Anionic and cationic composition of two different groups of acid wheys expressed as \% of dry matter [85].

\begin{tabular}{lcc}
\hline Fabrication : & $\begin{array}{c}\text { Pâte fraîche } \\
\left(\mathrm{g} \cdot 100 \mathrm{~g}^{-1} \mathrm{MS}\right)\end{array}$ & $\begin{array}{c}\text { Pâte molle } \\
\left(\mathrm{g} \cdot 100 \mathrm{~g}^{-1} \mathrm{MS}\right)\end{array}$ \\
\hline Potassium & $2,74 \pm 0,29$ & $3,10 \pm 0,19$ \\
Calcium & $1,29 \pm 0,28$ & $2,06 \pm 0,61$ \\
Sodium & $1,14 \pm 0,15$ & $1,11 \pm 0,25$ \\
Magnesium & $0,13 \pm 0,01$ & $0,19 \pm 0,03$ \\
Ammonium & $0,13 \pm 0,12$ & $0,23 \pm 0,11$ \\
Chlorure & $1,99 \pm 0,25$ & $2,15 \pm 0,08$ \\
Phosphate & $1,38 \pm 0,19$ & $3,78 \pm 1,50$ \\
Sulphate & $0,14 \pm 0,04$ & $0,15 \pm 0,02$ \\
Citrate & $2,21 \pm 0,33$ & $2,29 \pm 0,62$ \\
Lactate & $1,84 \pm 0,39$ & $5,82 \pm 2,48$ \\
Acétate & $0,16 \pm 0,03$ & $0,43 \pm 0,26$ \\
\hline
\end{tabular}

l'acidité des lactosérums acides varient fortement au cours de l'étape d'égouttage des fromages. Ces observations permettent de conclure qu'à chaque type de fromage et à chaque étape de fabrication est associé un lactosérum. De plus l'émergence de nouvelles technologies de fractionnement et de concentration comme l'ultrafiltration a permis 
l'apparition de nouveaux dérivés comme les perméats. Leur composition est fonction de nombreux paramètres comme la matière première ultrafiltrée, le type de membrane et le taux de concentration. L'extrait sec de ces produits est de $50-57 \mathrm{~g} \cdot \mathrm{kg}^{-1}$ dont 80 à $85 \%$ de lactose et 8 à $10 \%$ de cendres [16, $61,90]$.

\section{SÉCHAGE DES LACTOSÉRUMS ET DÉRIVÉS}

Il existe 2 grands types de procédés de séchage : le séchoir à cylindres ou le séchage par atomisation. Bien que la première méthode soit la moins coûteuse, cette technique peut causer, en raison de l'effet thermique, des dommages indésirables dans la plupart des applications fonctionnelles des produits de lactosérum. Elle n'est donc pratiquement plus utilisée sauf pour des applications particulières nécessitant un traitement thermique intensif comme la production de poudre de lait à forte teneur en matière grasse libre ou à forte réaction de Maillard pour l'industrie chocolatière [19]. Le séchage de concentré de lactosérum par atomisation est devenu la méthode d'obtention des poudres la plus utilisée [70]. Une fois séchées, les poudres sont stockées et expédiées.

\section{1. Étapes du procédé}

La transformation des lactosérums liquides en poudre implique la combinaison de plusieurs étapes unitaires : pré-traitement (déminéralisation, pasteurisation et ultrafiltration), concentration par évaporation sous vide (ESV), pré-cristallisation, séchage par atomisation, post-cristallisation, et séchage en lit fluidisé.

La concentration par évaporation sous vide du lactosérum permet de passer d'un extrait sec de 6,5\% à 50-60\%. L'énergie nécessaire est apportée sous forme de vapeur à pression réduite. Pour réduire les coûts de fonctionnement, les évaporateurs « modernes » comportent plusieurs effets avec thermocompression de la vapeur.
Un « flash cooler » ou refroidissement par détente en sortie d'ESV permet de refroidir rapidement le concentré (de 40 à $30{ }^{\circ} \mathrm{C}$ par exemple) et de démarrer la cristallisation qui se poursuivra en cuve. Selon le type de cristallisation (pré ou postséchage), la forme des cristaux de lactose obtenus varie, respectivement « tomahawk » et forme en aiguille [72]. Le produit précristallisé est ensuite séché par atomisation. La technique actuelle est l'atomisation en tour deux temps ou tour trois temps (multiple effet) [96]. Le séchage deux temps consiste à limiter le séchage par atomisation au profit d'un procédé à temps de séjour plus long, et donc plus proche de l'équilibre thermodynamique. Le produit à la sortie de l'unité de séchage par atomisation aura l'humidité maximale compatible avec une évacuation continue et un maintien de conditions d'exploitation acceptables. Ceci entraîne une sensible diminution de la température d'air de sortie, et permet également une augmentation de la température d'air d'entrée ; le produit plus humide, sera moins sensible au choc thermique. Le séchage final pour obtenir l'humidité résiduelle requise a lieu dans un lit fluidisé externe (ou vibro-fluidiseur) dont les débits d'air et températures sont adaptés à la préservation de la qualité du produit. Le principe du séchage deux temps indiquait clairement la voie à suivre pour réduire le coût du séchage et améliorer les performances et la qualité des produits déshydratés : transférer la plus grande partie possible de la phase dite «d'atomisation » à la phase dite de «fluidisation». La limite est le contact produit humide avec les parois de l'unité, contact inévitable compte tenu de la turbulence interne nécessaire à l'échange thermique. L'élimination de cette limite a été la naissance de la tour de séchage trois temps ou multiple effet. En effet, faute de pouvoir éliminer les parois de l'unité de séchage par atomisation, les équipementiers ont cherché à éviter tout contact entre celles-ci et le produit humide en stabilisant et séchant ce dernier dans un lit fluidisé interne (ou lit statique) à l'unité [48, 58, 


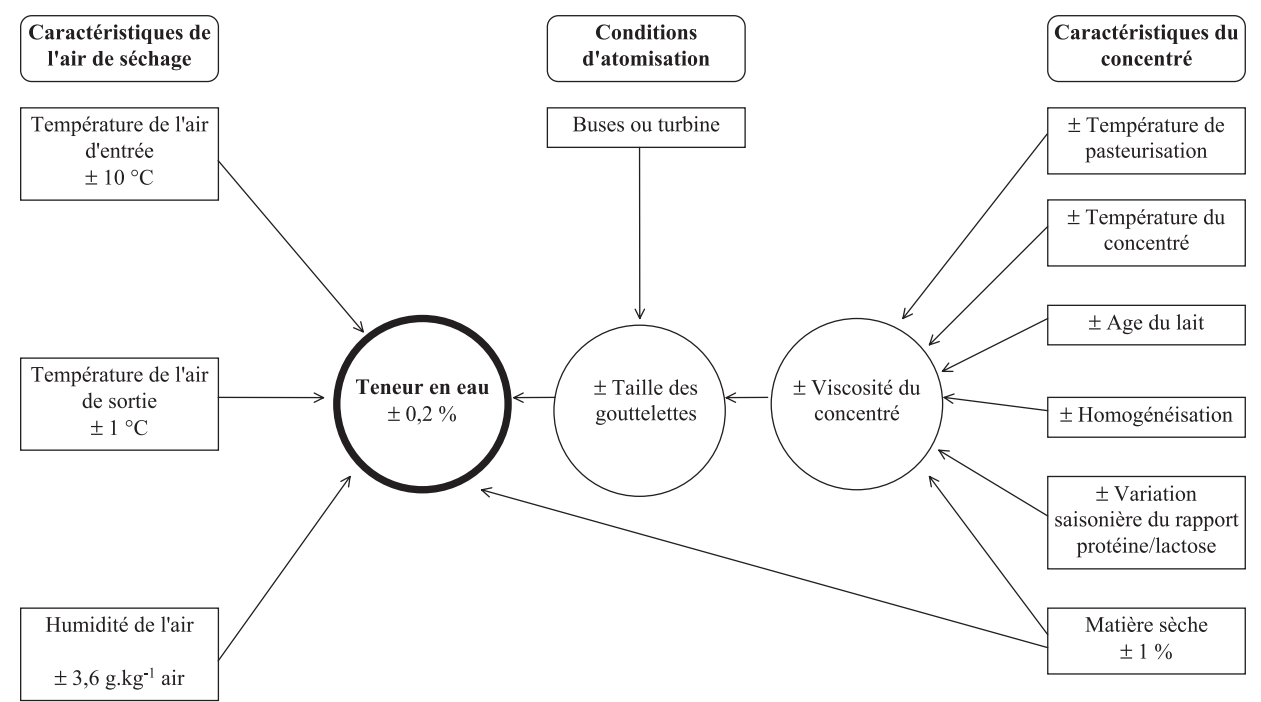

Figure 2. Influence des paramètres technologiques et physico-chimiques sur la teneur en eau d'une poudre laitière [69].

Figure 2. Influence of technological and physico-chemical parameters on the moisture of a dairy powder [69].

$70,96]$. Le couplage de la technique d'atomisation à la technique de fluidisation interne et/ou externe a ainsi permis d'améliorer la qualité des poudres par rapport aux séchages en un temps.

\subsection{Importance de la disponibilité et de l'activité de l'eau}

La disponibilité de l'eau est un facteur primordial pour le séchage des poudres, en particulier au travers de son effet sur la cristallisation du lactose. L'activité de l'eau $\left(\mathrm{a}_{\mathrm{w}}\right)$ est directement liée à l'humidité relative et influence fortement les propriétés physiques des produits (viscosité et transition vitreuse).

\subsubsection{Activité de l'eau et humidité relative}

Généralement, l'eau totale contenue dans un produit se divise en deux catégories : l'eau libre pouvant être extraite facilement [56] et l'eau liée subdivisée en plusieurs fractions selon son degré d'interaction avec la matière sèche [14]. La teneur en eau, exprimée en grammes d'eau pour $100 \mathrm{~g}$ de matière sèche, est une grandeur quantitative difficile à déterminer étant donné les états variés sous lesquels l'eau existe. Les principales méthodes de dosage de l'eau ont été répertoriées par Loisel [56] et les normes spécifiques aux produits alimentaires ont été établies par Multon et al. [64]. L'ensemble des facteurs qui ont une influence sur la teneur en eau d'une poudre de lait est récapitulé sur la figure 2 [69]. Cette figure montre que la teneur en eau d'une poudre de lait varie en fonction des paramètres thermodynamiques de l'air de séchage, des conditions de pulvérisation et des caractéristiques physico-chimiques du concentré avant séchage.

$L ' a_{w}$ exprime quant à elle la disponibilité de l'eau dans les produits et donc son aptitude à participer aux réactions comme solvant ou réactif. Il est donc nécessaire de quantifier cette $a_{w}$ dont la vitesse de dégradation des aliments dépend fortement (Fig. 3). 


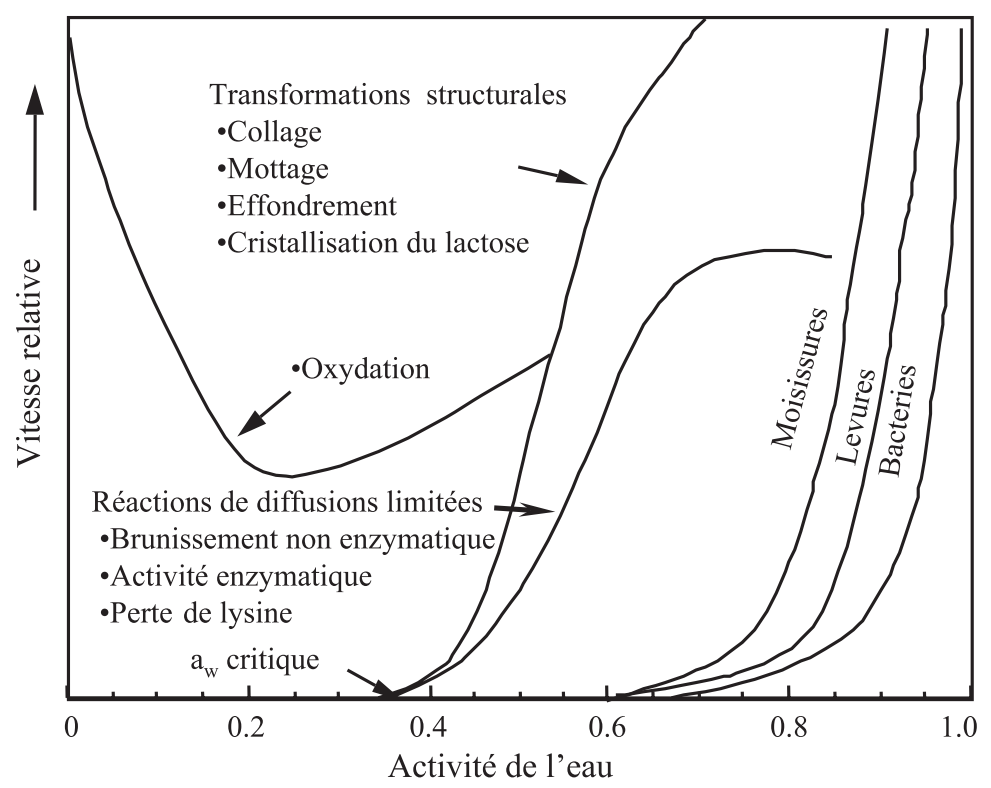

Figure 3. Vitesse de dégradation des aliments en fonction de l'activité de l'eau [12, 50].

Figure 3. Stability map of food products as affected by water activity $[12,50]$

Les produits se conservent mieux pour une $\mathrm{a}_{\mathrm{w}}$ de $0,2[12,50]$. L'état de l'eau dans les aliments a donc autant d'importance pour leur stabilité que la teneur en eau totale.

L'a $\mathrm{a}_{\mathrm{w}}$ d'un produit est une donnée thermodynamique reliée à l'humidité relative (HR) de l'air en équilibre avec ce produit. À température fixée, l' $\mathrm{a}_{\mathrm{w}}$ est algébriquement égale à l'HR de l'air à l'équilibre avec le produit, divisée par $100\left(\mathrm{a}_{\mathrm{w}}=\mathrm{HR}(\%) /\right.$ 100). L'ouvrage de Le Meste et al. [54] et la revue de Hardy et al. [28] donnent une présentation détaillée de la notion d'activité de l'eau.

Il existe plusieurs méthodes qui permettent la détermination de la valeur de l' $\mathrm{a}_{\mathrm{w}}$ $[25,54]$. Toutes sont reliées à la mesure de l'hygrométrie de l'air en équilibre, en pression et en température, avec le produit [56]. Ainsi, un écart de température produitmilieu génère un transfert d'eau dû à une variation de pression de vapeur. En consé- quence, la mesure d' $\mathrm{a}_{\mathrm{w}}$ est associée à une valeur de température.

Il existe à l'équilibre thermodynamique avec l'atmosphère environnante, une relation entre l' $\mathrm{a}_{\mathrm{w}}$ et la teneur en eau du produit. Ce phénomène est décrit par les isothermes de sorption (Fig. 4) qui reflètent la capacité d'adsorption et de rétention de l'eau [56]. Ces isothermes sont fonctions de la température et des caractéristiques chimiques et physico-chimiques de l'aliment étudié. La quantité et la qualité de l'eau sorbée et la cinétique de sorption sont influencées par ces caractéristiques [49,50].

Les trois zones de l'isotherme représentent l'eau plus ou moins fortement liée aux macromolécules (Fig. 4). Chou et Morr [14] ont défini différents types de molécules d'eau associées aux macromolécules : de la plus liée à la plus libre. Les isothermes sont donc utilisés pour déterminer la teneur en eau dite idéale pour la bonne conservation 


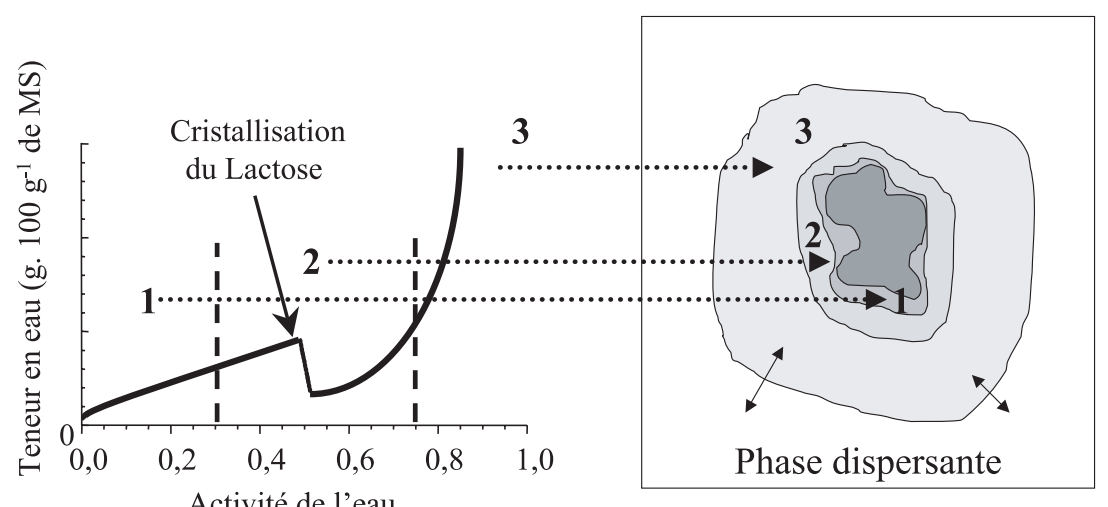

1: eau monocouche 2: eau multicouche 3: eau hydrodynamique

Figure 4. Isotherme d'adsorption d'une poudre de lait écrémé ou de lactosérum contenant initialement du lactose amorphe [3, 56, 77].

Figure 4. Sorption isotherm of skim milk or whey powder with amorphous lactose [3, 56, 77].

Tableau III. Pourcentage d'hygroscopicité ou de teneur en eau finale des différentes classes après exposition à un air à $80 \%$ d'HR $[18,31,71]$.

Table III. Hygroscopicity or final moisture content of different sections after air exposure at $80 \%$ RH $[18,31,71]$.

\begin{tabular}{lc}
\hline Classes & $\begin{array}{c}\text { Hygroscopicité ou teneur en humidité finale après } \\
\text { exposition à un air humide à } 80 \% \text { d'HR }(\%)\end{array}$ \\
\hline Non hygroscopique & $\leq 10,0$ \\
Peu hygroscopique & $10,1-15,0$ \\
Moyennement hygroscopique & $15,1-20,0$ \\
Hygroscopique & $20,1-25,0$ \\
Très hygroscopique & $>25,0$ \\
\hline
\end{tabular}

du produit. Ils permettent aussi de prédire le comportement d'un produit, d' $\mathrm{a}_{\mathrm{w}}$ défini, en fonction de l'HR de l'air en équilibre avec ce produit. Les notions d'a $a_{w}$ et de teneur en eau sont étroitement liées : plus la teneur en eau est élevée et plus l' $\mathrm{a}_{\mathrm{w}}$ tend vers l'activité de l'eau pure c'est-à-dire 1 .

Mais ces deux notions doivent aussi être mises en relation avec la composition du produit. En effet, l'affinité pour l'eau est fonction de nombreux paramètres intrinsèques au produit [56] : prédominance et accessibilité stérique des groupes polaires, état physique du produit (cristallin ou amor- phe), morphologie de surface, présence de pores et d'anfractuosités [49].

L'affinité d'un produit pour l'eau ou hygroscopicité a une influence sur les isothermes de sorption et donc sur l' $\mathrm{a}_{\mathrm{w}}$ et la teneur en eau. Ces quatre facteurs $\left(a_{w}\right.$, teneur en eau, composition et hygroscopicité) sont interdépendants. L'hygroscopicité est responsable de l'adsorption de la vapeur d'eau ambiante par des aliments, en opposition avec la désorption. L'application des méthodes de mesure de Haugaard Sorensen et al. [31] a permis de classer les poudres laitières en cinq catégories (Tab. III) 
en fonction du pourcentage d'hygroscopicité ou de la teneur en humidité finale après exposition à un air humide à $80 \%$ d'HR $[18,31,71]$.

\subsubsection{Relation entre disponibilité de l'eau et propriétés physiques de la poudre}

La mobilité de l'eau est fortement dépendante de la viscosité dynamique du produit. La viscosité dynamique newtonienne est définie par la relation : $\mu=\tau / \varepsilon$ où $\mu$ la viscosité en $\mathrm{Pa} \cdot \mathrm{s}$ est fonction de la contrainte de cisaillement $\tau$ en $\mathrm{Pa}$ et de la vitesse de cisaillement $\varepsilon$ en $\mathrm{s}^{-1}$. La plupart des produits alimentaires obéissent à des comportements de type non-newtoniens, ce qui conduit à parler de viscosité apparente pour des raisons de simplicité.

Dans une solution, la viscosité augmente parallèlement à la concentration en soluté, ce qui est associé à une baisse de la mobilité moléculaire et de la proportion de molécules d'eau de mobilité élevée [53]. Le comportement rhéologique d'un produit dépend également de la nature et de la structure de ses composants. L'augmentation de la viscosité peut être associée à la transition vitreuse car, en empêchant l'arrangement cristallin des molécules, elle permet la vitrification : les molécules n'ont pas le temps ni la possibilité d'adopter la conformation qui correspond au minimum d'énergie libre [52]. La transition vitreuse a lieu dans une zone de haute viscosité (de l'ordre de $10^{12}$ à $\left.10^{14} \mathrm{~Pa} \cdot \mathrm{s}\right)[52,76,77]$. L'équation WLF (Williams, Landel et Ferry) [108] permet d'estimer la viscosité du système dans un intervalle de température voisin mais supérieur à la température de transition vitreuse sous réserve de n'avoir aucune modification de structure ou de composition dans cet intervalle de température.

La transition vitreuse est un phénomène dont l'importance technologique est reconnue depuis de très nombreuses années que ce soit pour les substances minérales, organiques ou pour les produits alimentaires.
La transition vitreuse, caractérisée par une plage de températures (Tg), est un phénomène affectant les substances amorphes ou les zones amorphes de matériaux partiellement cristallisés [52].

À l'état solide amorphe, les molécules sont peu ordonnées et le système est dit dans un état vitreux : la matière est caractérisée par une haute viscosité interne. À la transition vitreuse, lors d'un réchauffement, le système passe de la forme vitreuse à l'état viscoélastique dans lequel la mobilité moléculaire est plus élevée [5]. Cette évolution peut également être obtenue, à température constante, par ajout de substances de faible poids moléculaire comme l'eau, on parle alors d'effet plastifiant (Fig. 5) [76, 77].

Ainsi, une variation de la température autour de la valeur de Tg s'accompagne d'une modification forte des propriétés mécaniques du matériau $[46,78]$. Bien que la transition vitreuse ait été exploitée pour prédire la stabilité de produits congelés ou déshydratés, la complexité et l'hétérogénéité des produits rendent les interprétations délicates. Les sucres, les protéines et la matière grasse sont les molécules alimentaires affectées par des transitions vitreuses [23, 79, 80].

Dans la pratique, la Tg est mesurée en suivant l'évolution du flux de chaleur spécifique en fonction de la température, généralement par l'analyse calorimétrique différentielle. Cette analyse permet de distinguer trois événements thermiques successifs lors du réchauffement d'un corps pur en structure amorphe [89] :

- à Tg, un changement de chaleur spécifique (endothermique) ;

- à la température de cristallisation, un changement d'état exothermique ; et

- à la température de fusion, un changement d'état endothermique.

Selon la localisation de la température du produit par rapport à la $\mathrm{Tg}$, il existera des variations au niveau de la nature et de l'amplitude des mouvements moléculaires 
Tg du lactose (----) Isotherme de sorption du lait écrémé (-)

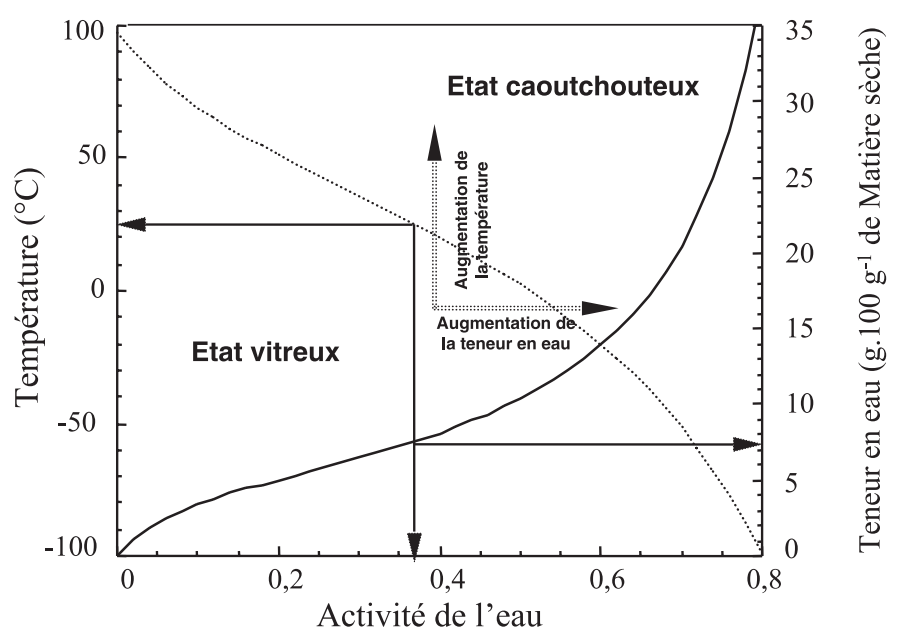

Figure 5. Diagramme de transition de phase et évolution de la teneur en eau et de la température de transition vitreuse du lactose en fonction de l'activité de l'eau d'une poudre de lait écrémé $[45,76]$.

Figure 5. Diagrammatic representation of phase transition and water adsorption isotherm and glass transition temperature of lactose for a skim milk powder [45, 76].

mais aussi au niveau de l'effet de la température sur la cinétique des évolutions. Pour une température inférieure à $\mathrm{Tg}$, seuls les mouvements locaux de faible amplitude, non coopératifs, sont possibles. Alors que pour une température supérieure à $\mathrm{Tg}$, des mouvements moléculaires de plus grande amplitude et coopératifs apparaissent. De plus, dans cette zone de températures $(\mathrm{T}>$ $\mathrm{Tg}$ ), la mobilité moléculaire est accentuée, favorisant ainsi de nombreuses réactions [52].

L'ajout de petites molécules comme l'eau abaisse la valeur de $\operatorname{Tg}[15,52]$. Ce mécanisme a pu être observé aussi bien pour du lactose amorphe que pour une poudre de lait écrémé $[45,55]$. Pour une $\mathrm{a}_{\mathrm{w}} \mathrm{de}$ 0,37 , la teneur en eau du lait est de 7,6 g pour $100 \mathrm{~g}$ de matière sèche et sa température de transition est de $24{ }^{\circ} \mathrm{C}$. En revanche, pour une $\mathrm{a}_{\mathrm{w}}$ de 0,2 la teneur en eau est voisine de $5 \mathrm{~g}$ pour $100 \mathrm{~g}$ de matière sèche et la température de transition passe à $50{ }^{\circ} \mathrm{C}$ (Fig. 5) [45]. Associées aux conditions opératoires, $\mathrm{a}_{\mathrm{w}}$ et $\mathrm{Tg}$ peuvent aider à formuler, à choisir et adapter le procédé de séchage. La disponibilité de l'eau dans une matrice alimentaire est donc fonction de différents facteurs : teneur en eau, composition en solutés, hygroscopicité, viscosité et Tg.

\subsection{Importance de la cristallisation du lactose}

\subsubsection{Cristallisation}

Le lactose, diholoside, est le produit de la condensation 1:4 entre une molécule d' $\alpha$ ou $\beta$ glucose et une molécule de $\beta$ galactose. Il existe 2 formes anomériques, lactose $\alpha(\mathrm{L} \alpha)$ et lactose $\beta$ (L $\beta)$, engendrées par une réaction de mutarotation et qui diffèrent par la configuration du groupe $\mathrm{OH}$ et de l'hydrogène fixés sur le carbone anomérique du glucose. Le lactose subit également d'autres réactions chimiques telles que l'isomérisation en présence de calcium [59] et l'hydrogénation [81] conduisant respectivement à la formation du lactulose et du lactitol [82]. Le lactose possède plusieurs sites chimiquement réactifs incluant la liaison 1-4 entre glucose et galactose, 
Tableau IV. Conditions d'obtention des différentes formes du lactose [104, 107].

Table IV. Lactose modifications [104, 107].

\begin{tabular}{lc}
\hline Formes & Conditions de formation \\
\hline$\alpha$-monohydratée & Solution sursaturée, $\mathrm{T}<93,5^{\circ} \mathrm{C}$ ou $\mathrm{a}_{\mathrm{w}} \geq 0,57$ \\
$\beta$-anhydre & Solution sursaturée, $\mathrm{T}>93,5^{\circ} \mathrm{C}$ ou $0,39<\mathrm{a}_{\mathrm{w}}<0,49$ \\
Amorphe & Séchage rapide atomisation ou cylindres ou $\mathrm{a}_{\mathrm{w}} \leq 0,39$ \\
$\alpha$-anhydre instable & Séchage sous vide, $100^{\circ} \mathrm{C}$ \\
$\alpha$-anhydre stable & Séchage à $150^{\circ} \mathrm{C}$ en présence de vapeur d'eau \\
& Solution éthanolique sursaturée \\
\hline
\end{tabular}

Tableau V. Propriétés physiques des formes $\mathrm{L} \alpha \mathrm{H}_{2} \mathrm{O}$ et $\mathrm{L} \beta$ du lactose [30].

Table V. Physical properties of $\alpha$-lactose monohydrate and $\beta$-lactose shape [30].

\begin{tabular}{|c|c|c|}
\hline Propriétés & Lactose $\alpha \mathrm{H}_{2} \mathrm{O}$ & Lactose $\beta$ \\
\hline Masse volumique $\left(\mathrm{g} \cdot \mathrm{1}^{-1}\right)$ & 1,544 & 1,590 \\
\hline Température de fusion $\left({ }^{\circ} \mathrm{C}\right)$ & 202 (dégradation) & 252 (dégradation) \\
\hline Pouvoir rotatoire $\left(^{\circ}\right)$ & +88 & +34 \\
\hline Chaleur de combustion $\left(\mathrm{J} \cdot \mathrm{g}^{-1}\right)$ & 16106 & 15465 \\
\hline Chaleur spécifique $\left(\mathrm{J} \cdot \mathrm{kg}^{-1} \cdot \mathrm{K}^{-1}\right)$ & 1251 & 1193 \\
\hline Chaleur de solubilisation $\left(\mathrm{J} \cdot \mathrm{g}^{-1}\right)$ & $-50,24$ & $-9,62$ \\
\hline Masse molaire $\left(\mathrm{g} \cdot \mathrm{mole}^{-1}\right)$ & 360,1 & 342,0 \\
\hline Solubilité initiale $\left(\mathrm{g} \cdot 100 \mathrm{~g}^{-1}\right)$ & 7,4 & 55,0 \\
\hline
\end{tabular}

le groupe aldéhyde du glucose, les fonctions hydroxyles et les liaisons carbone-carbone [109]. En fonction de la température et de l'état d'hydratation de la molécule, il y a formation de différents isomères : $\mathrm{L} \alpha \mathrm{H}_{2} \mathrm{O}$; $\mathrm{L} \beta$ anhydre ; $\mathrm{L} \alpha$ anhydre; $\mathrm{L} \beta \mathrm{H}_{2} \mathrm{O}$ (n'existe pas dans la nature); et $\mathrm{L}$ amorphe [47]. Dans une solution de lactose à $20^{\circ} \mathrm{C}$, les 2 formes ( $\alpha$ et $\beta$ ) coexistent dans les proportions respectives de 38 et $62 \%$. Cet équilibre, appelé mutarotation $[27,35,36$, 47], est indépendant de la forme initialement dissoute. La température l'influence selon la relation décrite par Roetman et Burma [74] avec $\beta / \alpha=(1.64-0.0027) \times$ $\mathrm{T}$, où $\mathrm{T}$ est la température en degré Kelvin et $\alpha$ et $\beta$ sont les proportions relatives des formes $\mathrm{L} \alpha$ et $\mathrm{L} \beta$ (\% massiques). Le tableau IV donne les principales formes cristallines du lactose et leur mode d'obtention [104, 107].

La mutarotation du lactose rend difficile l'étude des différentes formes cristallines du lactose en solution [21]. Elle est étroitement liée à la solubilité du lactose qui est faible et varie avec sa forme. Le L $\alpha$, moins soluble que le L $\beta$, cristallise donc en premier sous forme monohydratée. Pour maintenir l'équilibre des formes, du $\beta$ est converti en $\alpha$ qui cristallise au fur et à mesure de sa formation [101]. Les deux formes les plus fréquentes sont $\mathrm{L} \mathrm{H}_{2} \mathrm{O}$ et $\mathrm{L} \beta$, leurs propriétés sont données dans le tableau V. Ainsi, à $20^{\circ} \mathrm{C}, 7 \mathrm{~g}$ de L $\alpha$ peuvent être dissous dans $100 \mathrm{~g}$ d'eau contre $55 \mathrm{~g}$ de $\mathrm{L} \beta$ dans les mêmes conditions. $\mathrm{La}$ mutarotation établit un équilibre entre les deux formes et permet d'atteindre une solubilité finale de $17 \mathrm{~g}$ dans $100 \mathrm{~g}$ d'eau $[36,65]$. Le passage à la solubilité finale est contrôlée par la mutarotation du lactose $\alpha$ en $\beta$ plus soluble $[51,65,101]$. Selon Vuataz [105, 106], le diagramme de phase du lait entier (Fig. 6) montre que, pour éviter la cristallisation du lactose, la fenêtre de séchage est limitée d'une part par la courbe 


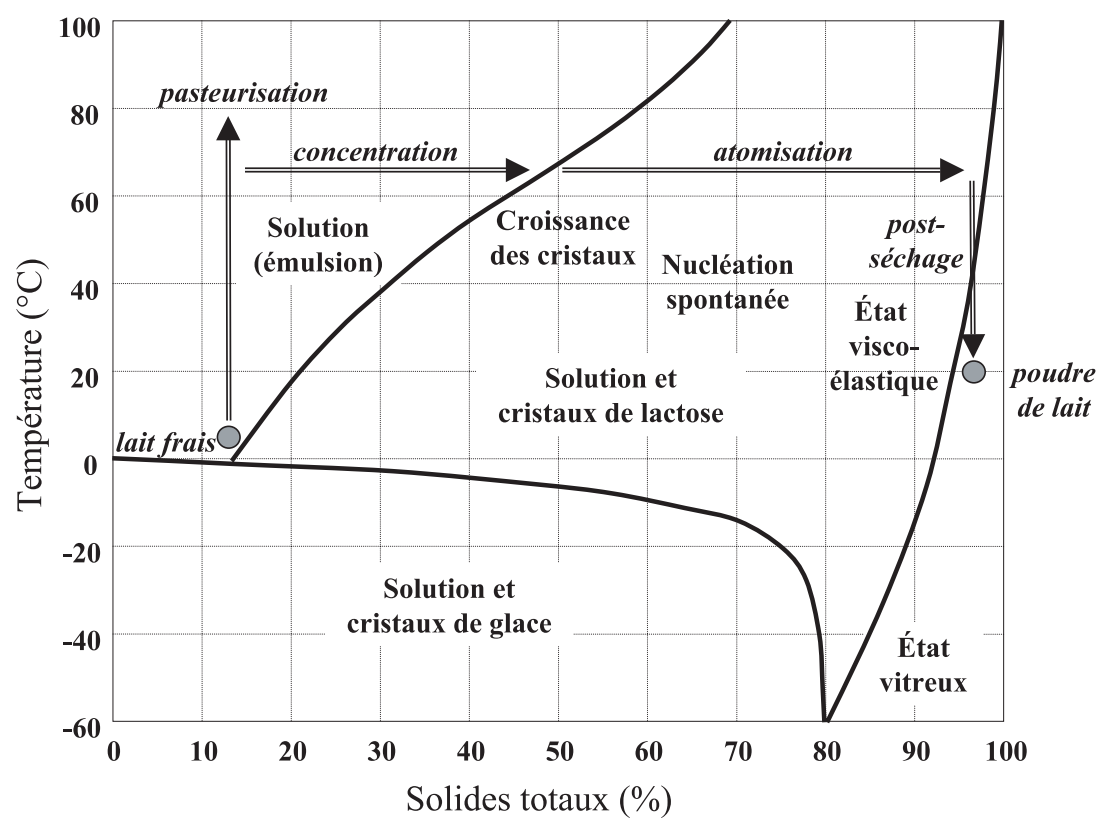

Figure 6. Diagramme de phase du lait entier (état du lactose et de l'eau) [105, 106].

Figure 6. Phase diagram of whole milk (state of lactose and water) $[105,106]$.

de solubilité (concentré), de pente relativement faible, et d'autre part par la courbe de transition vitreuse (poudre), de pente beaucoup plus élevée. Ce diagramme de phase se prête particulièrement bien à la description des différentes étapes du procédé de fabrication de la poudre de lait car il permet de positionner : la pasteurisation, la concentration, l'homogénéisation, le séchage par atomisation, le post-séchage et le postrefroidissement. Il peut également être adapté au lait écrémé ou encore au lactosérum car il ressort clairement [77] que le lactose conditionne l'allure générale du diagramme, ce qui permettrait ainsi d'envisager un « guide de travail rationnel » malgré la complexité physico-chimique de ces produits laitiers.

La solubilité du lactose diminue avec la température, il en découle une réaction de cristallisation, qui n'est pas immédiate : la solution reste d'abord dans un état sursaturé avant de commencer à cristalliser. $\mathrm{Ce}$ mécanisme est représenté par la courbe de solubilité du lactose (Fig. 7). À $50{ }^{\circ} \mathrm{C}$, la cristallisation n'intervient qu'à forte teneur de lactose dans l'eau, alors qu'à $20^{\circ} \mathrm{C}$, la cristallisation est amorcée à partir d'une teneur en lactose voisine de $40 \%$.

La cristallisation est produite à partir d'une solution sursaturée par deux processus simultanés de nucléation et de croissance. Elle débute par la formation de petits agrégats appelés germes ou nucléi [51]. L'étape de nucléation peut être homogène ou hétérogène.

Dans le premier cas, elle se produit spontanément dans une solution idéalement pure par la seule augmentation de la sursaturation. Les facteurs qui l'influencent sont ceux qui jouent sur la limite de la sursaturation et, plus particulièrement, la vitesse de production de la sursaturation, la température et l'énergie interfaciale cristal-solution. Mais il est possible d'y inclure l'agitation, la présence ou non d'impuretés, les chocs 


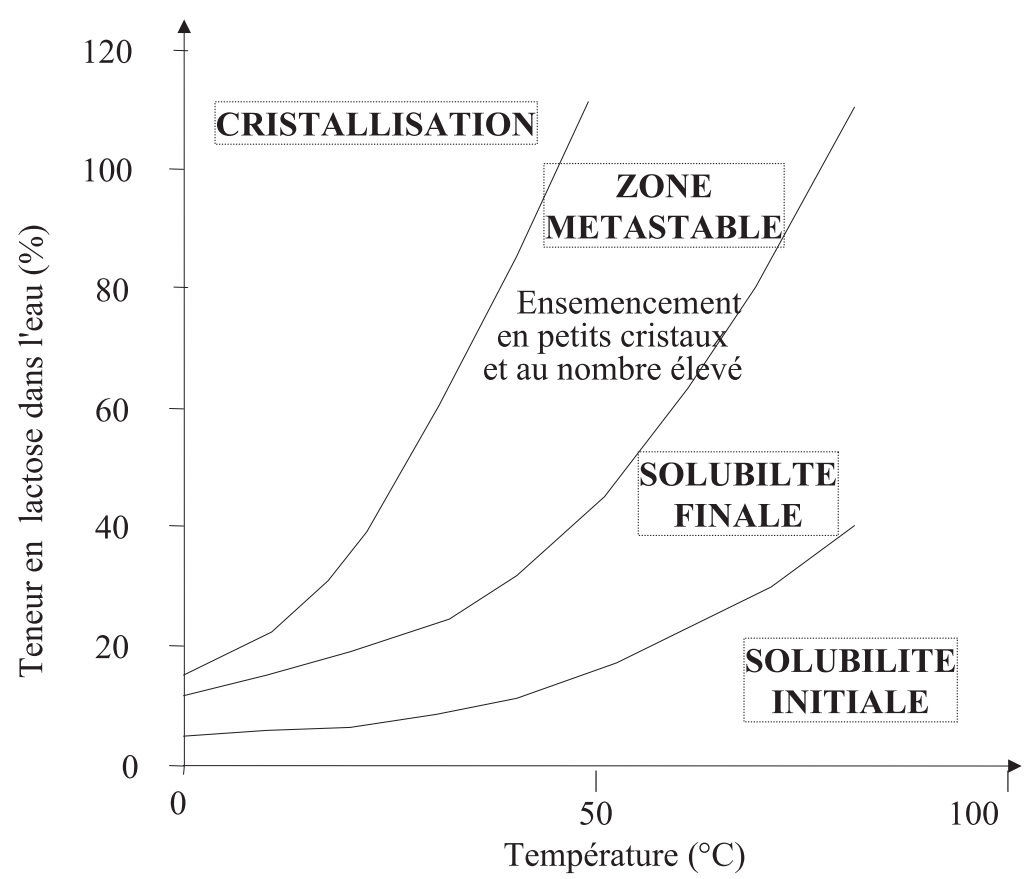

Figure 7. Courbes de solubilité et de cristallisation du lactose [51].

Figure 7. Curves of solubility and crystallization of lactose [51].

mécaniques et thermique, le $\mathrm{pH}$ et, d'une manière générale, l'histoire de la solution. Ainsi, lors d'une germination homogène, il faut se placer dans une zone de température compatible avec la nucléation et la croissance.

La nucléation hétérogène correspond à la réalité expérimentale. Elle est provoquée par des impuretés ou tout simplement par contact avec le récipient.

Une fois formés, les nucléi peuvent crô̂tre. La croissance résulte de deux étapes : la diffusion et la réaction de surface. Elle est donc influencée par tous les éléments qui jouent sur ces 2 réactions (agitation, température, sursaturation, viscosité et impuretés). Dans le cas de poudres amorphes, la recristallisation hétérogène du produit peut se produire lors du stockage par augmentation de la température ou de l'humidité (Fig. 4). La rupture brutale de l'isotherme de sorption observée dans le cas d'une poudre de lactosérum s'explique par un changement morphologique du lactose consécutif à la cristallisation de la forme amorphe initiale [3]. L'objectif recherché lors de la cristallisation est d'obtenir un produit peu hygroscopique, cristallisé essentiellement sous la

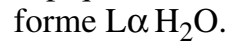

Différentes méthodes d'analyse ont permis de différencier qualitativement, et pour certaines quantitativement [73], les formes de lactose présentes. La diffraction des rayons $\mathrm{X}$ a permis d'identifier et de quantifier les formes $\alpha$ et $\beta$ [22]. Par calorimétrie différentielle à balayage, il est possible de quantifier la proportion de lactose amorphe présent [75] et donc, par différence, d'estimer la teneur en lactose cristallisé. L'enthalpie de cristallisation varie proportionnellement avec le pourcentage du lactose amorphe présent. L'enthalpie évolue de 5 à $95 \mathrm{~J} \cdot \mathrm{g}^{-1}$ lorsque ce pourcentage passe de 5 à $100 \%$ [89]. 


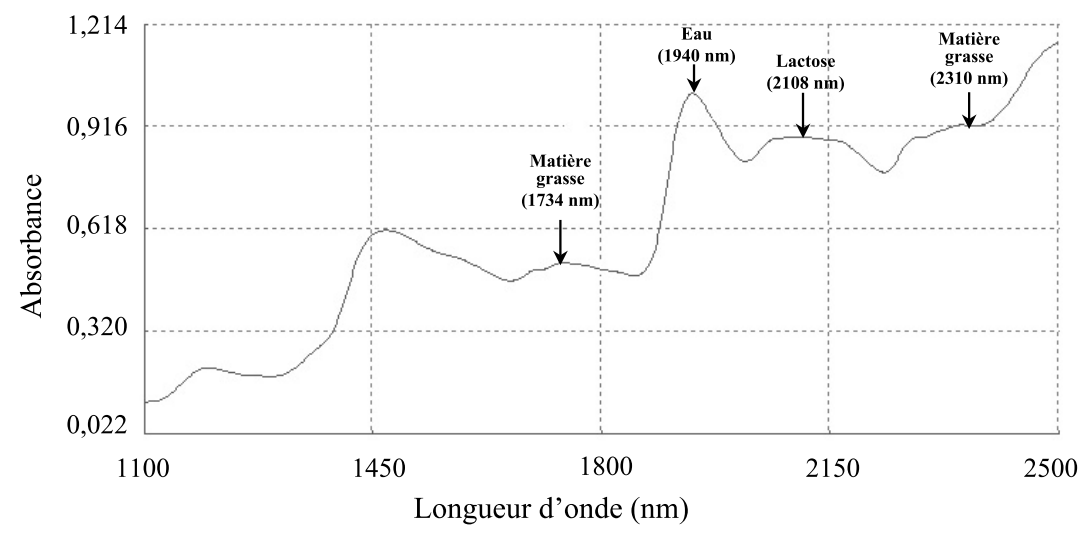

Figure 8. Spectre proche infra-rouge d'une poudre de lait écrémé [104].

Figure 8. Near infrared spectrum of a skim milk powder [104].

Le proche infra rouge (Fig. 8) est également très utilisé car il permet de différencier clairement les deux formes du lactose [104]. Une nouvelle méthode de détermination de la teneur en humidité totale par étuvage sous vide permettant de calculer le degré de cristallisation du lactose sous forme monohydratée a été proposée récemment [88].

\subsubsection{Paramètres qui influencent la cristallisation du lactose}

\subsubsection{Propriétés physiques}

La vitesse de croissance des cristaux augmente avec le degré de sursaturation de la solution [40, 98, 99]. Une élévation de l'extrait sec ou de la teneur en lactose dans un lactosérum ou dans un perméat d'ultrafiltration augmente le degré de sursaturation. Une forte viscosité est en revanche un frein à la formation de cristaux du fait d'une faible mobilité moléculaire.

Les conditions de refroidissement jouent sur la taille des cristaux : lorsqu'il est lent les cristaux sont gros [100]. Ainsi, l'ensemencement dans ces conditions permet aux cristaux de se développer complètement en prisme de forme complexe ou tomahawk. L'ensemencement se fait avec de petits cristaux en grande quantité pour avoir une grande surface de réaction et donc le maximum de cristallisation [2, 71]. La vitesse d'écoulement ou d'agitation doit être mâ̂trisée de manière à renouveler la solution mère au contact de la surface des cristaux et permettre leur croissance par l'apport de nouvelles molécules de lactose.

Le lactose pour cristalliser a besoin d'eau disponible (pour passer de la forme vitreuse à la forme cristalline) et par conséquent la réaction est influencée par :

- L'hygroscopicité du produit qui dépend essentiellement de sa composition. Selon la composition en minéraux, protéines, acide lactique, la cristallisation est ralentie ou inhibée. De nombreux auteurs ont étudié cette influence $[4,26,41,60,66,67,83,93$, 94, 103]. Dans la plupart des cas, les études ont été menées sur des solutions modèles et non sur des lactosérums. Les interactions entre les différents constituants ne sont pas prises en compte.

- La viscosité qui a un effet direct sur la mobilité moléculaire de l'eau et des constituants. La viscosité est par ailleurs ellemême fortement influencée par divers facteurs comme le $\mathrm{pH}$ de la solution et le taux de dénaturation des protéines $[6,17,63]$.

$-L ' a_{w}$ qui est reliée à la teneur en eau et à la $\mathrm{Tg}$ [23]. Lorsque la différence $\mathrm{T}-\mathrm{Tg}$ 
augmente, le taux et la vitesse de cristallisation sont accélérés.

- La mutarotation qui est dépendante des changements de la température ou encore de l'acidité et de la concentration en sels. Cela peut être l'étape limitante de la cristallisation dans les concentrés [18].

Pour optimiser la cristallisation, il est nécessaire de maîtriser le procédé et le produit. En pratique, le concentré est refroidi lentement pour avoir le meilleur compromis entre la vitesse de cristallisation qui est proportionnelle à la température et le taux de cristallisation qui est lui inversement proportionnel à la température [95].

\subsubsection{Constituants}

La teneur en minéraux et en protéines influence le processus de cristallisation du lactose. L'effet des minéraux réside dans la capacité à se complexer au lactose réduisant ainsi sa solubilité et par voie de conséquence sa cristallisation [32-34, 43, 97, 98]. Mais, étant donné la complexité et la diversité des lactosérums, il est difficile d'établir de véritables relations entre les différents constituants et les effets sur la cristallisation du lactose. C'est pourquoi, un grand nombre d'auteurs ont étudié ces interactions dans des solutions modèles de lactose supplémentées ou non avec l'additif à étudier. Ces expériences ont permis d'observer l'effet de ces composés sur la croissance des cristaux dans une solution sursaturée en lactose. L'ensemble des résultats est récapitulé dans le tableau VI. Les résultats diffèrent en raison des conditions expérimentales (température, $\mathrm{pH}$ et degré de sursaturation) qui varient selon les auteurs [40, 98, 99].

Les données du tableau VI illustrent le comportement des minéraux en solution de lactose mais pas dans un milieu complexe comme les lactosérums. En effet ce type d'expérimentation ne prend pas en compte les interactions possibles entre les constituants ce qui peut modifier le comportement d'un élément minéral. Le $\mathrm{pH}$ du lactosérum peut influencer la forme et le comportement des minéraux. Nickerson et Moore [66, 97] précisent qu'un $\mathrm{pH}$ acide favorise la cristallisation, mais ces conditions ne reflètent pas la réalité dans les produits alimentaires. Delaveau et Jelen [17] ont montré qu'une variation du $\mathrm{pH}$ (vers la zone alcaline) dans un lactosérum acide ou doux provoque une baisse de solubilité du phosphate de calcium et une augmentation de la viscosité. Cela a pour conséquence une baisse de la mobilité moléculaire nécessaire au mécanisme de cristallisation d'ou une baisse de la croissance des cristaux. Aussi, un lactosérum contenant des protéines dénaturées, à un $\mathrm{pH}$ proche du $\mathrm{pHi}$ des protéines, montre une croissance des cristaux plus faible et une viscosité plus élevée [13]. La viscosité et par conséquent la cristallisation sont donc influencées par les degrés de dénaturation et d'hydratation des protéines. Si le degré d'hydratation des protéines diminue, l'eau relarguée est disponible pour la cristallisation du lactose [63].

\section{PROBLÈMES LIÉS AU SÉCHAGE DES LACTOSÉRUMS ET ORIGINES}

Le collage en tour et de mottage sont les deux principaux problèmes rencontrés lors du séchage des lactosérums et leurs dérivés. Ces problèmes sont liés d'une manière relativement complexe aux différents facteurs décrits ci-dessus et à leurs interactions.

\subsection{Collage}

Dans certaines conditions, lors du séchage par atomisation, la poudre qui n'est pas totalement déshydratée, peut coller aux surfaces métalliques chaudes de l'installation et former un dépôt. Ce comportement connu comme le phénomène de thermoplasticité ou collage, limite la technique au niveau des températures d'air et de la matière sèche du produit $[23,37]$.

La plastification thermique ou par exposition à de hautes humidités d'une poudre 
Tableau VI. Effet de quelques substances organiques et minérales sur la cristallisation du lactose. Table VI. Effect of some organic and mineral substances on the lactose crystallization.

\begin{tabular}{|c|c|c|c|}
\hline Composés & Effet sur la cristallisation & & Auteurs \\
\hline Lactose $\beta$ & $\begin{array}{l}\text { * interaction avec certaines faces des cristaux et inhibition } \\
\text { de leur croissance } \\
\text { * ajout de lactose favorise la sursaturation et donc } \\
\text { la croissance des cristaux }\end{array}$ & - & $\begin{array}{c}{[60]} \\
{[66,67]}\end{array}$ \\
\hline Riboflavine & $\begin{array}{l}\text { * pas d'effet sur la cristallisation à sa teneur naturelle } \\
\text { dans les sérums }\end{array}$ & 0 & $\begin{array}{c}{[60,93,} \\
94]\end{array}$ \\
\hline $\mathrm{LiCl}$ & $\begin{array}{l}\text { * réorganisation du réseau de l'eau par le lithium ce qui } \\
\text { favorise la croissance des cristaux de lactose }\end{array}$ & + & [5] \\
\hline $\mathrm{NaCl}$ et $\mathrm{MgCl}_{2}$ & * pas d'effet sur la cristallisation & 0 & [93] \\
\hline $\mathrm{KCl}$ & $\begin{array}{l}\text { * effet sur la vitesse de cristallisation en fonction } \\
\text { de sa concentration }\end{array}$ & \pm & $\begin{array}{c}{[40,41,} \\
93]\end{array}$ \\
\hline Galactosyllactose & $\begin{array}{l}\text { * empêche la cristallisation en s'adsorbant à la surface } \\
\text { de certaines faces des cristaux }\end{array}$ & - & [94] \\
\hline $\mathrm{CaCl}_{2}$ & $\begin{array}{l}\text { * selon sa concentration il favorise ou retarde la croissance } \\
\text { des cristaux } \\
\text { * formation d'un précipité de phosphate de calcium } \\
\text { à la surface des cristaux ce qui inhibe la croissance }\end{array}$ & \pm & $\begin{array}{l}{[94]} \\
{[5]}\end{array}$ \\
\hline \multirow[t]{2}{*}{ Lactate } & $\begin{array}{l}\text { * quelque soit sa forme il accélère la cristallisation } \\
\text { * le lactate de calcium favorise légèrement la croissance } \\
\text { des cristaux }\end{array}$ & $\begin{array}{l}+ \\
+\end{array}$ & $\begin{array}{c}{[93]} \\
{[5,94]}\end{array}$ \\
\hline & $\begin{array}{l}\text { * sous forme acide lactique effet inhibiteur de la croissance } \\
\text { des cristaux }\end{array}$ & - & [67] \\
\hline \multirow[t]{2}{*}{ Phosphate } & $\begin{array}{l}* \mathrm{~K}_{2} \mathrm{PO}_{4} \text { : retarde la cristallisation } \\
* \text { sels de phosphate acide ou alcalin favorise la cristallisation }\end{array}$ & + & $\begin{array}{l}{[40,41]} \\
{[5,103]}\end{array}$ \\
\hline & $\begin{array}{l}\text { * selon le pH possibilité de former du lactose phosphate qui } \\
\text { inhibe la croissance des cristaux }\end{array}$ & - & {$[94,102]$} \\
\hline
\end{tabular}

amorphe (non cristallisée) génère une chute de la viscosité à la surface des particules selon le mécanisme de transition vitreuse. Le résultat est le collage interparticules ou au niveau de la paroi [20]. Les produits collants sont sensibles aux conditions de séchage. Ainsi une légère variation de quelques degrés provoque le collage, ce qui rend l'étape de séchage très délicate. Le collage a des conséquences économiques importantes notamment par l'immobilisation de l'installation et la prolongation du temps de nettoyage.

Le collage est un phénomène complexe qui n'est pas seulement influencé par le caractère plastifiant. La cristallisation peut 


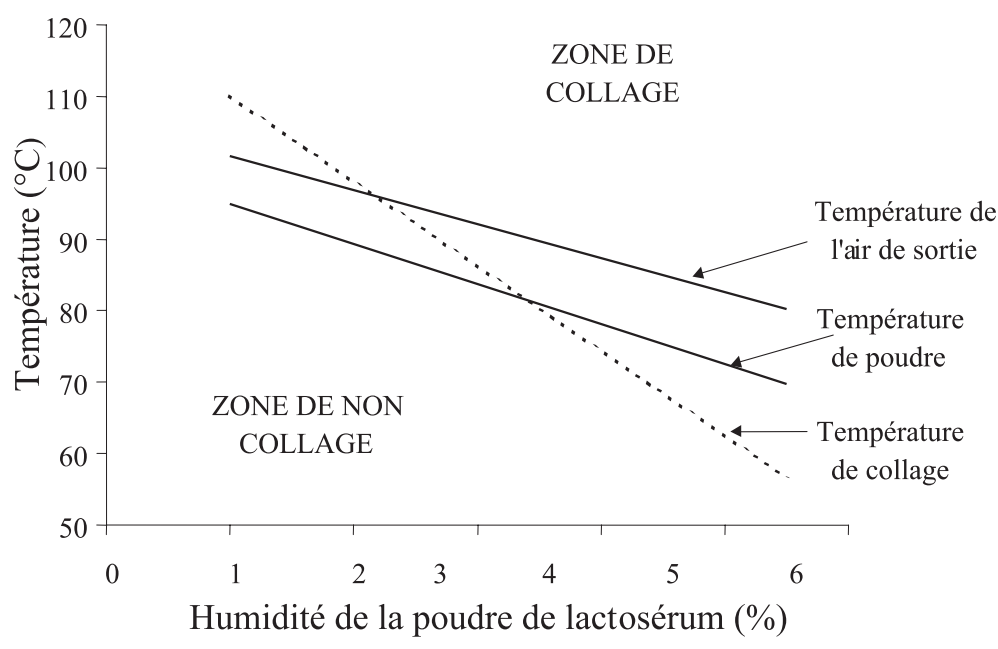

Figure 9. Influence de l'humidité sur la température de collage en fonction de la température de l'air de sortie et de la température de la poudre de lactosérum [37].

Figure 9. Dependence of sticking temperature on whey powder moisture, whey powder temperature and air drying temperature [37].

Tableau VII. Paramètres de séchage des produits laitiers [57, 58].

Table VII. Drying parameters of dairy products [57, 58].

\begin{tabular}{lccc}
\hline Produit & Extrait Sec initial $(\%)$ & $\begin{array}{c}\text { Température }\left({ }^{\circ} \mathrm{C}\right) \\
\text { air d'entrée }\end{array}$ & $\begin{array}{c}\text { Température }\left({ }^{\circ} \mathrm{C}\right) \\
\text { air de sortie }\end{array}$ \\
\hline Lait entier & $47-50$ & $175-240$ & $70-90$ \\
Lait écrémé & $47-52$ & $175-240$ & $75-90$ \\
Lactosérum & $40-60$ & $180-250$ & $80-95$ \\
\hline
\end{tabular}

jouer un rôle majeur. Un lactosérum concentré ne peut pas être séché directement après évaporation sans causer du collage en tour. Pour l'éviter, le lactose, caractérisé par une faible solubilité, est cristallisé sous forme $\alpha$ monohydratée $\left(\mathrm{L} \mathrm{H}_{2} \mathrm{O}\right)$, non hygroscopique. Le concentré est ensuite séché en tour réglée de manière à éviter l'adhésion aux parois. Des exemples de réglages de températures d'entrée et de sortie sont donnés dans le tableau VII.

Les températures d'air de sortie doivent être maintenues en dessous de $100{ }^{\circ} \mathrm{C}$ pour éviter la transformation du lactose $\mathrm{L} \mathrm{H}_{2} \mathrm{O}$ en lactose amorphe, qui peut s'agglomérer et coller aux parois. Sachant que l'humi- dité d'une poudre est gouvernée par son $\mathrm{a}_{\mathrm{w}}$ et donc par l'HR de l'air en équilibre avec la poudre, les facteurs positifs sur le séchage sont : la diminution de la température et de l'humidité de l'air d'entrée, l'augmentation de l'extrait sec du produit et l'utilisation du système de séchage à 2 ou 3 temps [37, 95, 96].

La figure 9 représente l'évolution de la température de collage en fonction de l'humidité, elle permet l'estimation des conditions opératoires, sous lesquelles il ne se produit pas de collage [37]. À titre d'exemple, pour une poudre ayant une humidité de $1 \%$, la température de l'air de sortie peut atteindre $100^{\circ} \mathrm{C}$ sans risque de 
Tableau VIII. Propriétés physiques des sucres et comportement face au collage au cours de l'opération de séchage [4].

Table VIII. Relationship between physical properties of carbohydrates and sticking during drying [4].

\begin{tabular}{lccccc}
\hline Sucres & $\begin{array}{c}\text { Hygroscopicité } \\
\text { relative à } \mathrm{a}_{\mathrm{w}}<0,5\end{array}$ & $\begin{array}{c}\text { Point de fusion } \\
\left({ }^{\circ} \mathrm{C}\right)\end{array}$ & $\begin{array}{c}\text { Solubilité approximative, } \\
\text { dans eau } 60^{\circ} \mathrm{C} \\
(\%, \mathrm{w} / \mathrm{w})\end{array}$ & $\begin{array}{c}\mathrm{Tg} \\
\left({ }^{\circ} \mathrm{C}\right)\end{array}$ & Collage \\
\hline Lactose & + & 223 & 35 & 101 & + \\
Maltose & ++ & 165 & 52 & 87 & ++ \\
Saccharose & +++ & 186 & 71 & 62 & +++ \\
Glucose & +++++ & 146 & 72 & 31 & ++++ \\
Fructose & ++++++ & 105 & 89 & 5 & +++++ \\
\hline
\end{tabular}

collage. En revanche, le collage intervient même si cette température est abaissée de $20{ }^{\circ} \mathrm{C}$ dans le cas d'une poudre à humidité de $5 \%$. Une tour fonctionne avec une marge de sécurité mais si les conditions varient, la température du produit devient supérieure à celle provoquant le collage : le produit colle aux parois de la tour. Cette situation est favorisée par l'augmentation de la température de sortie, la concentration du lactosérum ou encore l'humidité de l'air d'entrée [37, 42].

D'autres facteurs, ne relevant pas des conditions opératoires du procédé, doivent être pris en considération pour éviter le collage. Hynd [37] a montré que la température de collage dépend de la composition de la poudre et surtout des teneurs en acide lactique, en lactose amorphe et en eau. Lorsque la teneur en acide lactique augmente de $16 \%$, la température seuil de collage baisse de 98 à $57{ }^{\circ} \mathrm{C}$ rendant le produit plus sensible. En revanche, une baisse de la proportion en lactose amorphe de 55 à $20 \%$ déplace la température seuil de collage de 60 à $78^{\circ} \mathrm{C}$. Cette relation permet de guider la conduite du procédé pour éviter le collage. Ainsi, lorsque la température seuil de collage diminue sensiblement, il est conseillé de diminuer la température de l'air d'entrée et donc de la particule ainsi que le débit de produit à sécher tout en maintenant constante l'hygrométrie relative de l'air de sortie.
Le passage de la forme amorphe à la forme cristallisée diminue la proportion de composés hygroscopiques et améliore le séchage du produit. La nature du sucre présent ainsi que ses propriétés physiques (hygroscopicité, solubilité et $\mathrm{Tg}$ ) influencent le collage, ce que montre le tableau VIII [5, 81]. Une élévation de température de l'air de sortie augmente la solubilité des sucres, ce qui favorise le retour à la forme amorphe. Il faudra ainsi tenir compte de ce facteur pour maîtriser le collage.

Récemment, un grand nombre d'auteurs ont utilisé la température de transition vitreuse (Tg) comme indicateur du collage [5, 7, 8, $46,78,81,92]$. La température de transition vitreuse caractérise en effet le passage de la forme vitreuse à viscoélastique, étape intermédiaire avant la cristallisation (Fig. 10).

La cinétique de collage est fonction de la différence entre la température du produit et Tg selon Labuza cité par Bhandari [5] (Tab. VIII). Selon cet auteur, il faut se placer à une température inférieure à $\mathrm{Tg}+$ $20{ }^{\circ} \mathrm{C}$ pour éviter ce problème tout en tenant compte de l'évolution du produit au cours du procédé. Des méthodes d'ajout d'additifs comme les maltodextrines ont pour but d'augmenter $\mathrm{Tg}$ et donc le rendement en poudre [23].

Les particules collent si suffisamment de liquide «coule» entre elles afin de construire des ponts interparticulaires. À l'état vitreux, la viscosité élevée nécessite 

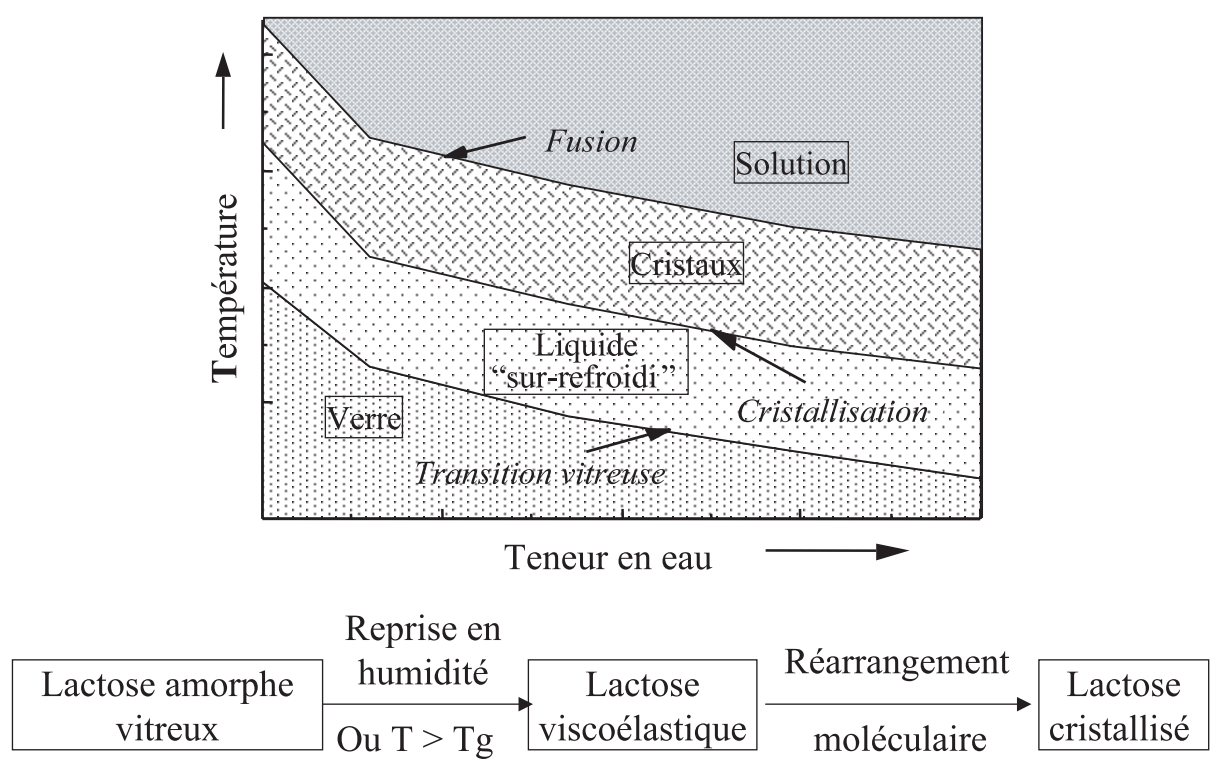

Figure 10. Dynamique de changement d'état du lactose amorphe [76].

Figure 10. Effect of water content on glass transition and melting temperature of amorphous lactose [76].

un temps de contact élevé pour permettre leur formation. Alors qu'au-dessus de Tg, la viscosité diminue et avec elle, le temps de contact nécessaire à leur formation. Downton et al. [20] et Dumoulin et Bimbenet [23] estiment qu'un temps de contact de 1 à $10 \mathrm{~s}$ (pour une viscosité inférieure à $10^{6}-10^{8} \mathrm{~Pa} \cdot \mathrm{s}$ ) est suffisant pour générer le collage.

La qualité du produit obtenu est fonction d'un grand nombre de paramètres et notamment de la qualité de la matière première et du procédé appliqué. Ainsi, une poudre de lait écrémé est de qualité différente selon qu'elle est séchée par atomisation ou par cylindres chauffants [62]. La figure 11 regroupe l'ensemble des propriétés des poudres de lait $[58,69]$.

\subsection{Mottage}

Les problèmes associés aux produits riches en sucre ne se limitent pas à l'étape de séchage mais aussi à leur conservation. Le mottage, ou prise en masse du produit, est une altération qui se produit lors du stockage ou du transport. Ce mécanisme transforme une poudre de faible humidité et d'écoulement libre en un produit solide aggloméré et collant. Une définition précise est très difficile à donner car le mottage implique divers mécanismes et il se déroule en plusieurs étapes [1]. La première étape initialise le processus par formation de ponts liquides interparticulaires. Ces ponts, qui nécessitent une faible viscosité, se solidifient par la suite sous diverses conditions (température, hygrométrie et durée du stockage). La formation des ponts peut être due à l'adsorption d'eau, la fusion de composés, des réactions chimiques qui produisent de l'eau comme le brunissement non enzymatique ou encore à l'eau relarguée lors de la cristallisation de sucres amorphes.

Le mécanisme le plus fréquent de mottage est la plastification due à l'adsorption 


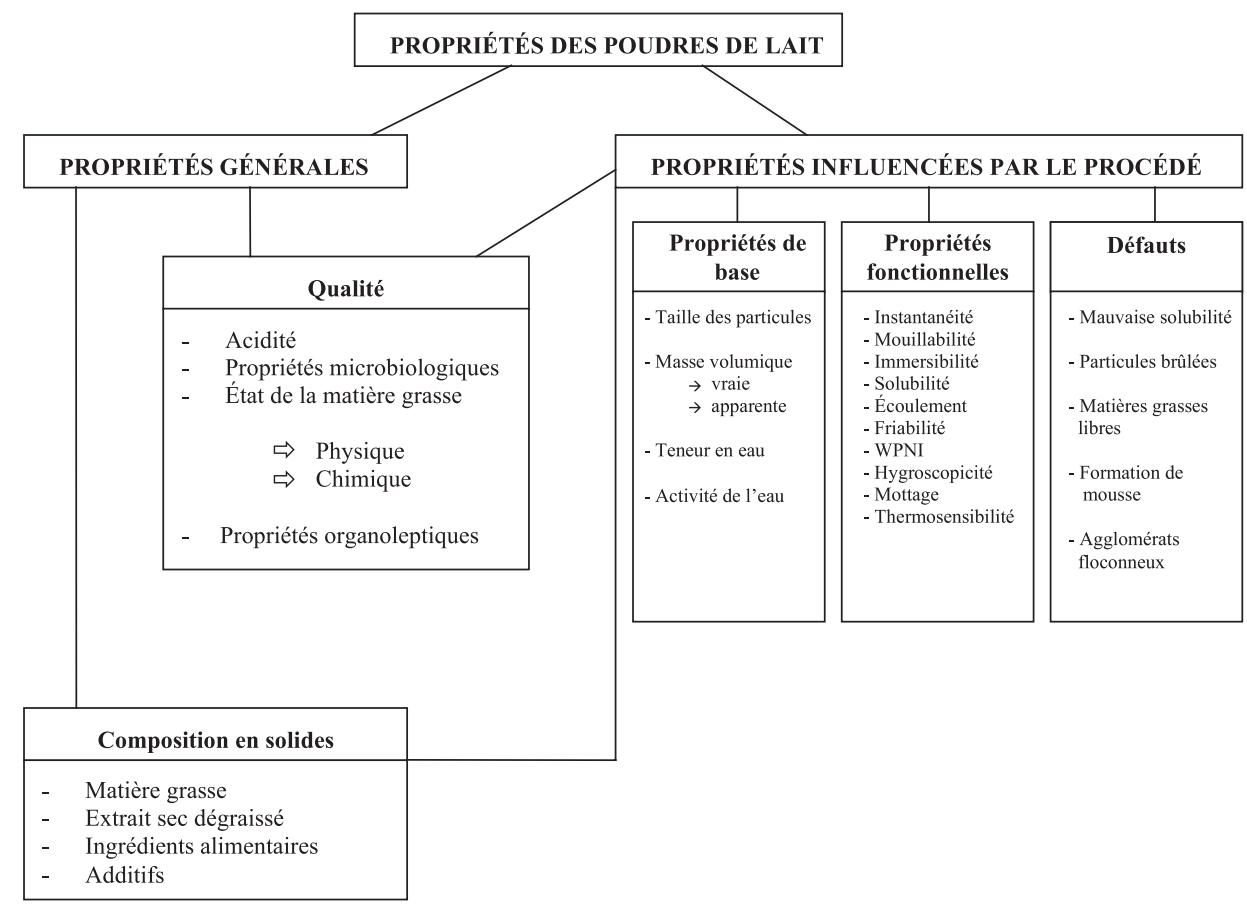

Figure 11. Principales propriétés des poudres de lait [57, 69].

Figure 11. Properties of milk powders [57, 69].

d'eau qui génère la fusion des particules. C'est pourquoi, de nombreux auteurs étudient la stabilité physique et physico-chimique des poudres riches en sucre vis-à-vis de l'eau. Toute diminution de la viscosité suite à un passage du sucre de l'état vitreux à l'état viscoélastique augmente la mobilité de l'eau et favorise ainsi la formation de ponts interparticulaires. Ainsi, une poudre exposée à de hautes humidités ou températures de stockage a tendance à s'agglomérer spontanément [24]. Le mottage peut se limiter à la formation de petits amas ou agglomérats ou conduire à la solidification de la poudre entière. Ceci conduit à une perte de fonctionnalité et de qualité du produit. Parmi les solutions proposées pour réduire ou éviter le mottage, on peut citer la formulation du produit (ajout «d'antimottant »), le choix du procédé et de l'équipement, les conditions opératoires du séchage et stockage, et plus particulièrement les paramètres $\mathrm{a}_{\mathrm{w}}, \mathrm{Tg}$, viscosité et humidité $[1,15]$. Une déminéralisation préalable, réduisant les taux de sodium, de calcium et d'acide lactique permet de limiter ces phénomènes [39].

La conservation de la poudre dépendra essentiellement de ces facteurs qui vont jouer sur la transition lactose amorphe-lactose cristallisé. Ainsi, $\mathrm{a}_{\mathrm{w}}$, humidité relative et température sont des paramètres dont il faudra tenir compte lors du stockage des poudres [84]. La cristallisation débute à $\mathrm{Tg}$ et a lieu à un taux et à une vitesse déterminée par $\mathrm{T}-\mathrm{Tg}$ [81]. L'humidité qui influe sur cette réaction est l'un des paramètres critique du mottage ; il peut être contrôlé en maîtrisant l'hygrométrie de l'installation ou par l'utilisation d'agents « antimottants » et d'emballage adéquats [1].

L'humidité du produit est reliée à sa capacité à fixer de l'eau c'est-à-dire à son 

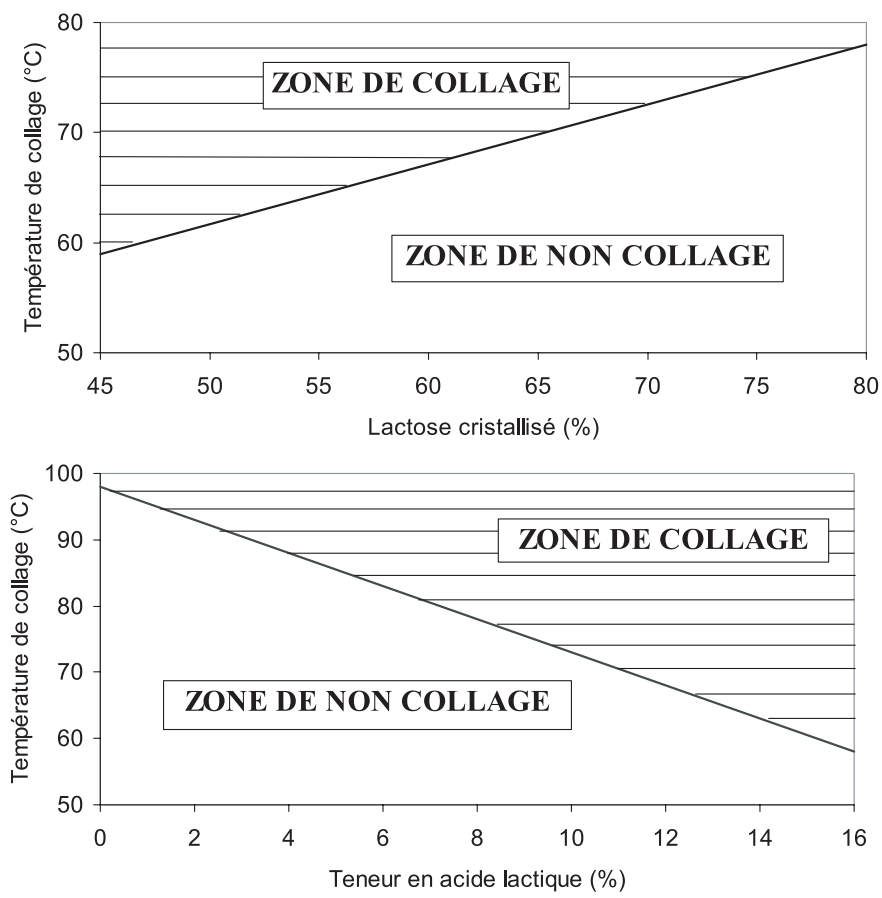

Figure 12. Collage des poudres de lactosérum en relation avec le degré de cristallisation et la teneur en acide lactique [71].

Figure 12. Sticking of whey powder as a function of crystallization degree and lactic acid content [71].

Tableau IX. Comparaison des propriétés de diverses poudres de lactosérum [71].

Table IX. Comparison of the properties of different whey powders [71].

\begin{tabular}{lcccc}
\hline Propriétés (\%) & Poudre ordinaire & Poudre pré-cristallisée & $\begin{array}{c}\text { Poudre non mottante } \\
\text { (séchage 2 temps) }\end{array}$ & $\begin{array}{c}\text { Poudre non mottante } \\
\text { (séchage } 3 \text { temps) }\end{array}$ \\
\hline Eau totale & $3-4$ & $3-5$ & $5-6$ & $4-5,5$ \\
Eau libre & $3-4$ & $1-2$ & $1,5-2,5$ & $1-2$ \\
Mottage & 100 & $40-100$ & $0-5$ & $0-5$ \\
Cristallisation & $0-10$ & $50-75$ & $75-85$ & $85-95$ \\
\hline
\end{tabular}

hygroscopicité. Le mottage peut donc être aussi causé par [91] :

- des constituants hygroscopiques : protéines, minéraux, acide lactique ;

- des constituants qui deviennent collants par reprise d'eau et/ou augmentation de la température ambiante (lactose amorphe).

L'eau absorbée et celle relarguée par cristallisation de composés amorphes sont responsables du collage en tour et du mottage en silo. L'obtention de poudre de lactosérum non mottante passe par la cristallisation du lactose avant séchage comme le montre le tableau IX.

Plus le degré de cristallisation est élevé, plus le risque de mottage est faible (Fig. 12). Ce risque augmente en même temps que la teneur en eau ou en acide lactique (Fig. 12) [71]. 
Collage et mottage sont deux phénomènes proches, dépendants de paramètres relatifs au procédé et au produit. L'un des moyens de les résoudre est d'introduire une étape de cristallisation avant séchage : elle améliore la qualité des poudres obtenues mais diminue aussi les coûts de production [9].

\section{CONCLUSION}

Il existe une grande diversité de lactosérums dont l'hétérogénéité de composition est due aux origines et au passé technologique du produit. La valeur nutritionnelle du lactosérum peut conduire ces produits à trouver de plus en plus d'applications dans l'alimentation humaine comme substitut du lait écrémé, et comme source de protéine et de lactose. La nécessité de développer des techniques de traitement doux est impérative pour mettre à disposition du marché des produits de qualité [38]. Ainsi, l'objectif de la déshydratation est de stabiliser ces produits afin de pouvoir les stocker et les transporter sans qu'ils se dégradent. Le séchage par atomisation est devenu, depuis plus de 50 ans, la méthode qui s'est imposée et qui a su évoluer. Le manque de données sur l'influence du procédé et des caractéristiques du concentré explique pourquoi la maîtrise de ce procédé s'est faite de manière empirique. Aujourd'hui, du fait de la diversité et de la complexité des produits traités, il est nécessaire d'adopter une démarche rigoureuse basée sur des approches thermodynamiques et physico-chimiques [86]. En effet, si les effets des divers facteurs technologiques sur le séchage des produits riches en sucres sont maintenant bien connus, la connaissance et la maîtrise de l'ensemble des mécanismes impliqués sont loin d'être acquises.

Collage et mottage sont complexes et sont influencés par un ensemble de paramètres interdépendants. Les données disponibles montrent que la maitrise de la cristallisation du lactose est primordiale et constitue un moyen de prévenir ces risques. Le cas d'un lactosérum délactosé appuie cette hypothèse : son séchage est beaucoup plus difficile car il est pauvre en lactose donc cristallisera peu, et il contient de fortes teneurs en protéines (plus ou moins dénaturées) et en minéraux (rapportés à l'extrait sec). La cristallisation permet d'obtenir un produit peu hygroscopique et donc peu sensible aux problèmes de collage et de mottage. Les notions d' $\mathrm{a}_{\mathrm{w}}$, de teneur en eau, de $\mathrm{Tg}$, de viscosité associées aux conditions opératoires et à la composition du produit sont utilisées pour prédire son comportement lors du traitement. L'obtention d'une poudre stable et de bonne qualité passe par une meilleure connaissance de ces facteurs et de leurs effets couplés.

\section{RÉFÉRENCES}

[1] Aguilera J.M., Del Valle J.M., Karel M., Caking phenomena in amorphous food powder, Trends Food Sci. Technol. 6 (1995) 149-155.

[2] Alais C., Sciences du lait. Principes des Techniques Laitières, SEPAIC, Paris, $4^{\mathrm{e}}$ edn., 1984.

[3] Berlin E., Anderson B.A., Pallansch M.J., Effect of temperature on water vapor sorption by dried milk powders, J. Dairy Sci. 53 (1970) 146-149.

[4] Bhandari B.R., Datta N., Howes T., Problems associated with spray drying of sugar-rich foods, Dry. Technol. 15 (1997) 671-684.

[5] Bhargava A., Jelen P., Lactose solubility and crystal growth as affected by mineral impureties, J. Food Sci. 61 (1996) 180-184.

[6] Buma T.J., Viscosity and density of concentred lactose solutions and of concentred cheese whey, Neth. Milk Dairy J. 34 (1980) 65-68.

[7] Busin L., Mortazavi F., Bimbenet J.J., Étude expérimentale du séchage par atomisation de solutions sucrées, Ind. Alim. Agric. 112 (1995) 485-489.

[8] Busin L., Buisson P., Bimbenet J.J., Notion de transition vitreuse appliquée au séchage par pulvérisation de solutions glucidiques, Sci. Aliments 16 (1996) 443-459.

[9] Caric M., Concentrated and dried dairy products, in: Hui Y.H. (Ed.), Dairy Science and Technology Handbook, 2. Product Manufacturing, VCH Publishers Inc, New York, USA, 1993, pp. 257-300. 
[10] Centre National Interprofessionnel de l'Économie Laitière, L'économie laitière en chiffres, CNIEL, Paris, France, 2001.

[11] Chaput G., Problèmes techniques et économiques posés par le stockage, le transport, la concentration et le séchage du lactosérum, Tech. Lait. 952 (1981) 25-28.

[12] Cheftel J.C., Cheftel H., L'eau, in: Cheftel J.C., Cheftel H. (Eds.), Introduction à la Biochimie et à la Technologie des Aliments, Tec. et Doc., Lavoisier, Paris, France, 1977, pp. 3-32.

[13] Chojnowski W., Dziuba J., Denaturation of whey proteins as influence by concentration, Milchwissenschaft 37 (1982) 476-478.

[14] Chou D.H., Morr C.V., Protein-water interactions and functional properties, J. Amer. Oil Chem. Soc. 56 (1979) 53-62.

[15] Chuy L.E., Labuza T.P., Caking and stickiness of dairy based food powder as related to glass transition, J. Food Sci. 59 (1994) 43-46.

[16] Coton S.G., The utilization of permeates from the ultrafiltration of whey and skim milk, J. Soc. Dairy Technol. 33 (1980) 8994.

[17] Delaveau J., Jelen P., Effect of pH on viscosity of sweet and acid wheys, J. Dairy Sci. 62 (1979) 1455-1457.

[18] De Vider J., Influence de la cristallisation du lactose dans le concentré de sérum sur l'hygroscopicité et l'agglomération de la poudre de sérum, Rev. Agric. 4 (1979) 953-975.

[19] De Witt J.N., Manuel de l'Enseignant sur le Lactosérum et les Produits de Lactosérum, $1^{\mathrm{e}}$ édn., European Whey Products Association, Bruxelles, Belgique, 2001.

[20] Dowton G.E., Flores-Luna J.L., King C.J., Mechanism of stickiness in hygroscopic, amorphous powder, Ind. Eng. Chem. Fundam. 21 (1982) 447.

[21] Drapier-Bêche N., Existence, caractérisation, analyses physico-chimiques et hypothèses de structure des composés moléculaires du lactose alpha/béta, Thèse INPL, Nancy, France, 1997.

[22] Drapier-Bêche N., Fanni J., Parmentier M., Vilasi M., Evaluation of lactose crystalline forms by nondestructive analysis, J. Dairy Sci. 80 (1997) 457-463.

[23] Dumoulin E., Bimbenet J.J., Mechanical, physical and chemical phenomena during food drying: consequences on properties of dried products, in: Drying'98, Proceedings of the 11th International Drying Symposium (IDS 98), Halkidiki, Greece, 1998, pp. 771-718.
[24] Elisée P., Influence de l'état de cristallisation du lactose et de l'hydratation sur les propriétés rhéologiques des poudres de lait écrémée, Thèse INPL, Nancy, France, 1992.

[25] Guilbert S., Morin P., Définition et mesure de l'activité de l'eau : revue des méthodes pratiques et théoriques, Lebensm.-Wiss. U.-Technol. 19 (1986) 395-400.

[26] Guu M.Y.K., Zall R.R., Lactose crystallization: effects of minerals and seeding, Process Biochem. 26 (1991) 167-172.

[27] Haase G., Nickerson T.A., Kinetic reactions of alpha and beta lactose, I. Mutarotation, J. Dairy Sci. 49 (1966) 127-132.

[28] Hardy J., Scher J., Banon S., Water activity and hydration of dairy powders, Lait 82 (2002) 441-452.

[29] Hargrove R.E., McDonough F.E., Lacroix D.E., Alford J.A., Production and properties of deproteinized whey powders, J. Dairy Sci. 59 (1976) 25-33.

[30] Harper W.J., Lactose and lactose derivatives, in: Zadow J.G. (Ed.), Whey and Lactose Processing, Elsevier Applied Science, London, England, 1992, pp. 317-360.

[31] Haugaard Sorensen I., Krag J., Pisecky J., Westergaard V., Méthodes d'analyses des produits laitiers déshydratés, Niro A/S, Copenhague, Danemark, 1978.

[32] Herrington B.L., Some physico-chemical properties of lactose. 2. Factors influencing the crystalline habit of lactose, J. Dairy Sci 17 (1934) 533-542.

[33] Herrington B.L., Some physico-chemical properties of lactose. 5. The influence of other substances upon the equilibrium rotation of lactose, J. Dairy Sci. 17 (1934) 701-707.

[34] Herrington B.L., Some physico-chemical properties of lactose. 6 . The solubility of lactose in salts solutions; the isolation of a compound of lactose and calcium chloride, J. Dairy Sci. 17 (1934) 805-814.

[35] Hudson C.S., Ueber die multirotation des milchzuckers, Z. Physik. Chem. 44 (1903) 487-502.

[36] Hudson C.S., The hydratation of milk sugar solution, J. Amer. Chem. Soc. 26 (1904) 1065-1082.

[37] Hynd J., Drying of whey, J. Soc. Dairy Technol. 33 (1980) 52-54.

[38] Imbert-Pontaven A., Étude de l'évolution de la composition des lactosérums au cours de leur conservation, Lait 568 (1977) 521-546.

[39] Jeantet R., Nanofiltration de liquides laitiers, Thèse ENSA, Rennes, France, 1995.

[40] Jelen P., Coulter S.T., Effects of supersaturation and temperature on the growth of 
lactose crystals, J. Food Sci. 38 (1973) 1182-1185.

[41] Jelen P., Coulter S.T., Effects of certains salts and other whey substances on the growth of lactose crystals, J. Food Sci. 38 (1973) 1186-1189.

[42] Jensen G.K., Oxlund J.K., Concentration and drying of whey and permeates, Bull. Int. Dairy Fed. 233 (1988) 4-20.

[43] Jensen O.G., Hanford Z.M., Supplee G.C., Equilibrium solutions of certain lactose salt mixtures, J. Dairy Sci. 23 (1940) 745.

[44] Josephson R.V., Rizvi S.S.H., Harper W.J., Compositional differences in whey systems, J. Food Sci. 40 (1975) 479-483.

[45] Jouppila K., Roos Y.H., Glass transition and crystallization in milk powders, J. Dairy Sci. 77 (1994) 2907-2915.

[46] Jouppila K., Kansikas J., Roos Y.H., Glass transition, water plasticization, and lactose crystallzation in skim milk powders, J. Dairy Sci. 80 (1994) 3152-3160.

[47] King N., The physical structure of dried milk, Dairy Sci. Abstr. 27 (1965) 91-104.

[48] Knipschildt M.E., Drying of milk and milk products, in: Robinson R.K. (Ed.), Modern Dairy Technology. Advances in Milk Processing, Elsevier, London, England, 1986, pp. 131-234.

[49] Labuza T.P., Sorption phenomena in foods, Food Technol. 22 (1968) 263-272.

[50] Labuza T.P., Sorption phenomena in foods: theorical and pratical aspects, in: Rha C.K. (Ed.), Theory, determination and control of physical properties of food materials, Reidel, Dordrecht, Germany, 1975, p. 197.

[51] Lamy J.P., La cristallisation du lactose, Rev. ENIL 106 (1986) 22-27.

[52] Le Meste M., Simatos D., La transition vitreuse : indices en technologie alimentaire, Ind. Alim. Agric. 107 (1990) 5-11.

[53] Le Meste M., Roudaut E.C., Simatos D., Colas B., Propriétés fonctionnelles de l'eau dans les aliments, Ind. Alim. Agric. 118 (2001) 21-28.

[54] Le Meste M., Lorient D., Simatos D., L'eau dans les Aliments, Tec. et Doc., Lavoisier, Paris, France, 2002.

[55] Lloyd R.J., Chen D.X., Hargreaves J.B., Glass transition and caking of spray-dried lactose, Int. J. Food Sci. Technol. 31 (1996) 305-311.

[56] Loisel C., Contribution à l'étude des propriétés rhéologiques de quelques poudres alimentaires, influence des conditions hygrométriques. Thèse universitaire, Nantes, France, 1988.
[57] Masters K., Spray Drying, Longman Scientific \& Technical and John Wiley \& Sons Inc., Essex, UK, 1991.

[58] Masters K., Spray Drying in Practice, SprayDryConsult International Aps, Charlottenlund, Danemark, 2002.

[59] Mendez A., Olano A., Lactulose: a review of some chemical properties and applications infant nutrition and medecine, Dairy Sci. Abstr. 41 (1979) 531-535.

[60] Michaels A.S., Van Kreveld A., Influences of additives on growth rates in lactose crystals, Neth. Milk Dairy J. 20 (1966) 163-181.

[61] Milanovic S., Caric M., Production and quality of demineralized permeate, concentrated permeate and permeate powder, Milchwissenschaft 45 (1990) 303-308.

[62] Missirian C., Costa P., Duprat J.C., Touron B., Agro-alimentaire : séchage sous vide en couche mince, Cah. Ing. 38 (1990) 12-18.

[63] Molder H.W., Lefkovitch L.P., Influence of $\mathrm{pH}$, casein, and whey protein denaturation on the composition, crystal size and yield of lactose from condensed whey, J. Dairy Sci. 69 (1986) 684-697.

[64] Multon J.L., Bizot H., Martin G., Eau (teneur, activité, absorption, propriétés fonctionnelles) humidités relatives, in: Deymié B., Multon J.L., Simon D. (Eds.), Techniques d'Analyse et de Contrôle dans les Industries Agro-alimentaires, 4, Analyse des Constituants Alimentaires, Tec. et Doc., Lavoisier, Paris, France, 1981, pp. 1-60.

[65] Nickerson T.A., Lactose chemistry: a review, J. Agric. Food Chem. Soc. 27 (1979) 672677.

[66] Nickerson T.A., Moore E.E., $\alpha$ lactose and crystallization rate, J. Dairy Sci. 57 (1973) 160-164.

[67] Nickerson T.A., Moore E.E., Factors influencing lactose crystallization, J. Dairy Sci. 57 (1974) 1315-1319.

[68] Patel R.S., Jayaprakasha H.M., Singh S., Recent advances in concentration and drying of whey, Indian Dairyman (1991) 417-421.

[69] Pisecky J., Technology of skimmed milk drying, J. Soc. Dairy Technol. 34 (1981) 57-62.

[70] Pisecky J., Technology advances in the production of spray dried milk, J. Soc. Dairy Technol. 38 (1985) 60-64.

[71] Pisecky J., Handbook of milk powder manufacture, Niro A/S, Copenhague, Danemark, 1997.

[72] Roetman K., Crystalline lactose and structure of spray-dried milk products as observed by scanning electron microscopy, Neth. Milk Dairy J. 33 (1979) 1-11. 
[73] Roetman K., Methods for the quantitative determination of crystalline lactose in milk products, Neth. Milk Dairy J. 35 (1981) 1-52.

[74] Roetman K., Buma T.J., Temperature dependence of the equilibrium $\beta / \alpha$ ratio of lactose in aqueous solution, Neth. Milk Dairy J. 28 (1974) 155-165.

[75] Roos K.D., Rapide determination of $\alpha$-lactose in whey powder by differential scanning calorimetry, J. Dairy Sci. 61 (1978) 255259.

[76] Roos Y.H., Water in milk products, in: Fox P.F. (Ed.), Advanced Dairy Chemistry Volume 3, Lactose, water, salts and vitamins, Chapman \& Hall, London, UK, 1997, pp. 303-346.

[77] Roos Y.H., Importance of glass transition and water activity to spray drying and stability of dairy powders, Lait 82 (2002) 475-484.

[78] Roos Y.H., Karel M., Plasticizing effect of water on thermal behavior and crystallization of amorphous food models, J. Food Sci. 56 (1991) 38-43.

[79] Roos Y.H., Karel M., Phase transitions of amorphous sucrose and frozen sucrose solutions, J. Food Sci. 56 (1991) 266-267.

[80] Roos Y.H., Karel M., Water and molecular weight effects on glass transitions in amorphous carbohydrates and carbohydrates solutions, J. Food Sci. 56 (1991) 1676-1681.

[81] Roos Y.H., Karel M., Crystallization of amorphous lactose, J. Food Sci. 57 (1992) 775-777.

[82] Saijonmea T., Heikonen M., Kreula M., Linko P., Preparation and characterisation of milk sugar alcohol, lactitol, Milchwissenschaft 33 (1978) 733-736.

[83] Saito Z., Lactose crystallization in commercial whey powders and in spray-dried lactose, Food Microstruct. 7 (1988) 75-81.

[84] Saltmarch M., Labuza T.P., Influence of relative humidity on the physicochemical state of lactose in spray-dried sweet whey powders, J. Food Sci. 45 (1980) 1231-1242.

[85] Saulnier F., Calco M., Humbert G., Linden G., Composition minérale et organique de différents lactosérums industriels, analysée par électrophorèse capillaire, Lait 76 (1996) 423-432.

[86] Schuck P., Appréhension des mécanismes de transfert d'eau lors du séchage par atomisation de bases protéiques laitières et lors de leur réhydratation. Effet de l'environnement glucidique et minéral, Thèse ENSA, Rennes, France, 1999.

[87] Schuck P., Spray drying of dairy products: state of the art, Lait 82 (2002) 375-382.
[88] Schuck P., Dolivet A., Lactose crystallization: determination of lactose $\alpha$ monohydrate in spray dried dairy products, Lait 82 (2002) 413-421.

[89] Senoussi A., Dumoulin E.D., Berk Z., Retention of diacetyl in milk during spraydrying and storage, J. Food Sci. 60 (1995) 894-897, 905.

[90] Shay L.K., Wegner G.H., Nonpolluting conversion of whey permeate to food yeast protein, J. Dairy Sci. 69 (1986) 676-683.

[91] Sienkiewicz T., Riedel C.L., Whey and Whey Utilization: Possibilities for Utilization in Agriculture and Foodstuffs Production, Verlag Th. Mann, Gelsenkirchenbuer, Germany, 1990.

[92] Slade L., Levine H., Ievolella J., Wang M., The glassy state phenomenon in application for the food industry: application of the food polymer science approach to structurefunction relationships of sucrose in cookie and cracker systems, J. Sci. Food Agric. 63 (1993) 133-176.

[93] Smart J.B., Effect of whey component on the rate of crystallization and solubility of $\alpha$ lactose monohydrate, N.Z. J. Dairy Sci. Technol. 23 (1988) 275-289.

[94] Smart J.B., Smith J.M., Effects of selected compounds on the rate of $\alpha$ lactose monohydrate crystallization, crystal yield and quality, Int. Dairy J. 1 (1991) 41-53.

[95] Sottiez P., Produits dérivés des fabrications fromagères, in: Luquet F.M. (Ed.), Lait et Produits Laitiers, Vol. 2, Tec. et Doc. Lavoisier, Paris, France, 1975, pp. 357-392.

[96] Sougnez M., L'évolution du séchage par atomisation, Chim. Mag. 1 (1983) 1-4.

[97] Swartz M.L., Bernhard R.A., Nickerson T.A., Interactions of metal ions with lactose, J. Food Sci. 43 (1978) 93-97.

[98] Thurlby J.A., Sitnai O., Lactose crystallization: investigation of some process alternatives, J. Food Sci. 41 (1976) 43-47.

[99] Twieg W.C., Nickerson T.A., Kinetics of lactose crystallization, J. Dairy Sci. 51 (1968) 1720-1724.

[100] Valle-Vega P., Nickerson T.A., Moore E.E., Gonzenbach M., Variability of growth of lactose crystals under commercial treatment, J. Dairy Sci. 60 (1977) 1544-1549.

[101] Veisseyre R., Technologie du lait, La Maison Rustique, Paris, 1975.

[102] Visser R.A., A natural crystal growth retarder in lactose, Neth. Milk Dairy J. 340 (1980) 255-275.

[103] Visser R.A., Crystal growth retarding of $\alpha$ lactose hydrate by sugar phosphate; a continued study, Neth. Milk Dairy J. 42 (1988) 449-468. 
[104] Vuataz G., Preservation of skim milk powders: role of water activity and temperature in lactose crystallization and lysine loss, in: Seow C.C. (Ed.), Food preservation by moisture control, Elsevier Applied Science, London, UK, 1988, pp. 73-101.

[105] Vuataz G., Prévention des transitions de phases dans les systèmes déshydratés pendant le traitement et le stockage, in: Les Rencontres Agoral (Ed.), Les Produits alimentaires et l'eau - L'eau dans l'Aliment - L'eau matière première, Tec. et Doc., Lavoisier, Paris, France, 1999, pp. 75-86.
[106] Vuataz G., The phase diagram of milk: a new tool for optimising the drying process, Lait 82 (2002) 485-500.

[107] Walstra P., Jeness R., Carbohydrates, in: Walstra P., Jeness R. (Eds.), Dairy Chemistry and Physics, J. Wiley et Sons, New York, USA, 1984, pp. 27-41.

[108] Williams M.L., Landel R.F., Ferry J.D., The temperature dependance of relaxation mechanisms in amorphous polymers and other glass-forming liquids, J. Amer. Chem. Soc. 77 (1955) 3701.

[109] Zadow J.G., Lactose: properties and uses, J. Dairy Sci. 67 (1984) 2654-2679. 